\title{
TWISTED TORSION INVARIANTS AND LINK CONCORDANCE
}

\author{
JAE CHOON CHA AND STEFAN FRIEDL
}

Dedicated to the memory of Des Sheiham

\begin{abstract}
The twisted torsion of a 3-manifold is well-known to be zero whenever the corresponding twisted Alexander module is non-torsion. Under mild extra assumptions we introduce a new twisted torsion invariant which is always non-zero. We show how this torsion invariant relates to the twisted intersection form of a bounding 4-manifold, generalizing a theorem of Milnor to the non-acyclic case. Using this result, we give new obstructions to 3-manifolds being homology cobordant and to links being concordant. These obstructions are sufficiently strong to detect that the Bing double of the figure eight knot is not slice.
\end{abstract}

\section{INTRODUCTION}

In this paper we introduce new obstructions to links being concordant, which are obtained from twisted torsion invariants. Recall that an $m$-component (oriented) link is an embedded ordered collection of $m$ disjoint (oriented) circles in $S^{3}$. Given an oriented link $L$ we denote by $-L$ the same link with the orientation of each component reversed. We say that two $m$-component oriented links $L_{0}=L_{0}^{1} \cup$ $\cdots \cup L_{0}^{m}$ and $L_{1}=L_{1}^{1} \cup \cdots \cup L_{1}^{m}$ are concordant if there exists a collection of $m$ disjoint, locally flat, oriented annuli $A_{1}, \ldots, A_{m}$ in $S^{3} \times[0,1]$ such that $\partial A_{i}=$ $L_{0}^{i} \times 0 \cup-L_{1}^{i} \times 1, i=1, \ldots, m$. We say that an $m$-component link is slice if it is concordant to the trivial $m$-component link. Equivalently a link is slice if it bounds $m$ disjoint locally flat disks in $D^{4}$.

Let $L \subset S^{3}$ be an oriented $m$-component link. Throughout this paper we write $E_{L}=S^{3} \backslash \nu L$, where $\nu L$ is a tubular neighborhood of $L$, and $\pi(L)=\pi_{1}\left(E_{L}\right)$. Let $\psi: H_{1}\left(E_{L}\right) \rightarrow H$ be a non-trivial homomorphism to a free abelian group and let $\alpha: \pi(L) \rightarrow \mathrm{GL}(R, k)$ be a representation with $R$ a subring of $\mathbb{C}$. We can then consider the twisted homology module $H_{1}\left(E_{L} ; R[H]^{k}\right)$ and we define

$$
\operatorname{rank}(L, \psi, \alpha)=\operatorname{rank}_{R[H]} H_{1}\left(E_{L} ; R[H]^{k}\right),
$$

where $\operatorname{rank}_{R[H]} A$ denotes the dimension of $A \otimes_{R[H]} Q(H)$ over the quotient field $Q(H)$ of the integral domain $R[H]$. If $\operatorname{rank}(L, \psi, \alpha)=0$, then it turns out that $E_{L}$ is $Q(H)^{k}$-acyclic, i.e., $H_{*}\left(E_{L} ; Q(H)^{k}\right)=0$, and we can define the torsion $\tau^{\alpha \otimes \psi}(L) \in Q(H)^{\times}$which is well-defined up to multiplication by an element of the form $\pm d h$ where $d \in \operatorname{det}(\alpha(\pi(L)))$ and $h \in H$. This invariant generalizes the invariants introduced by Reidemeister, Milnor and Turaev and later by Lin and Wada.

In general though $\operatorname{rank}(L, \psi, \alpha)$ will be non-zero, for example this is the case for any boundary link with at least two components (cf. Theorem 6.1) and the trivial representation. Suppose that $R \subset \mathbb{C}$ is closed under complex conjugation. In that case the ring $R[H]$ has a natural involution given by complex conjugation and $\bar{h}=h^{-1}$ for $h \in H$, this involution furthermore extends naturally to an involution

2000 Mathematics Subject Classification. 57M25, 57M27, 57N70.

Key words and phrases. Twisted Torsion, Homology Cobordism, Link Concordance. 
on $Q(H)$. Now suppose that $\alpha: \pi(L) \rightarrow \mathrm{GL}(R, k)$ is a unitary representation and suppose that $\psi$ is non-trivial on each meridian of $L$. Under these assumptions we define a torsion invariant

$$
\tau^{\alpha \otimes \psi}(L) \in Q(H)^{\times} / N(Q(H))
$$

even if $\operatorname{rank}(L, \psi, \alpha) \neq 0$. Here $N(Q(H))$ denotes the subgroup of norms of the multiplicative group $Q(H)^{\times}$, i.e., $N(Q(H))=\left\{ \pm q \bar{q} \mid q \in Q(H)^{\times}\right\}$. The torsion $\tau^{\alpha \otimes \psi}(L)$ viewed as an element in $Q(H)^{\times} / N(Q(H))$ is again well-defined up to multiplication by an element of the form $\pm d h$ where $d \in \operatorname{det}(\alpha(\pi(L)))$ and $h \in H$. The invariant $\tau^{\alpha \otimes \psi}(L)$ is the twisted version of an invariant first introduced by Turaev Tu86, Section 5.1].

Remark. If $K$ is a knot in $S^{3}, \psi: H_{1}\left(E_{K}\right) \rightarrow \mathbb{Z}$ is an isomorphism, and $\alpha: \pi(L) \rightarrow$ $\operatorname{GL}(\mathbb{Z}, 1)$ is the trivial representation, then $\operatorname{rank}(K, \psi, \alpha)=0$ and $\tau^{\alpha \otimes \psi}(K)=$ $\Delta_{K}(t) /(t-1)$ (cf. Tu01]). In general, if $\operatorname{rank}(L, \psi, \alpha)=0$, then $\tau^{\alpha \otimes \psi}(K)$ can be expressed in terms of twisted Alexander polynomials (cf. [KL99a and [FK06]). If $\alpha$ is the trivial representation and if $\operatorname{rank}(L, \psi, \alpha)>0$, then the torsion is related to the Alexander polynomial defined using $\operatorname{Tor}_{\mathbb{Z}[H]}\left(H_{1}\left(E_{L} ; \mathbb{Z}[H]\right)\right)$ (cf. [Tu86, Theorem 5.1.1]). We expect that a similar result also holds in the twisted case.

We can now state our sliceness obstruction. First, if $L$ is the $m$-component unlink in $S^{3}$ with meridians $\mu_{1}, \ldots, \mu_{m}$, then given $\alpha$ and $\psi$ as above we will show in Corollary 6.2 that $\operatorname{rank}(L, \psi, \alpha)=k(m-1)$ and

$$
\tau^{\alpha \otimes \psi}(L)= \pm d h \cdot \prod_{i=1}^{m} \operatorname{det}\left(\operatorname{id}-\psi\left(\mu_{i}\right) \alpha\left(\mu_{i}\right)\right)^{-1} \in Q(H)^{\times} / N(Q(H))
$$

with $d \in \operatorname{det}(\alpha(\pi(L)))$ and $h \in H$. The following theorem says that the torsion invariant of a slice link is given by the above expression if the representation factors through a $p$-group. This is a special case of our main result, Theorem 4.2, which is a more general result for link concordance.

Theorem 1.1. Let $L$ be an $m$-component oriented slice link. Let $R \subset \mathbb{C}$ be a subring closed under complex conjugation and let $\alpha: \pi(L) \rightarrow \mathrm{GL}(R, k)$ be a representation which factors through a finite group of prime power order. Let $\psi: H_{1}\left(E_{L}\right) \rightarrow H$ be an epimorphism onto a free abelian group which is non-trivial on each meridian of $L$. Then

$$
\operatorname{rank}(L, \psi, \alpha)=k(m-1)
$$

and

$$
\tau^{\alpha \otimes \psi}(L)= \pm d h \cdot \prod_{i=1}^{m} \operatorname{det}\left(\mathrm{id}-\psi\left(\mu_{i}\right) \alpha\left(\mu_{i}\right)\right)^{-1} \in Q(H)^{\times} / N(Q(H))
$$

for some $d \in \operatorname{det}(\alpha(\pi(L)))$ and $h \in H$, where $\mu_{1}, \ldots, \mu_{m}$ are meridians of $L$.

Remark. Let $K \subset S^{3}$ be a knot. Fox and Milnor [FM66] have shown that if $K$ is slice, then the untwisted Alexander polynomial of $K$ factors as $\pm t^{l} f(t) f\left(t^{-1}\right)$ for some polynomial $f(t)$ and some $l \in \mathbb{Z}$. It is well-known that for a knot all representations which factor through a $p$-group are necessarily abelian, in particular one can easily show that the sliceness obstruction of Theorem 1.1 reduces to the Fox-Milnor obstruction. On the other hand for knots Kirk and Livingston KL99a used twisted torsion corresponding to 'Casson-Gordon'-type representations to give sliceness obstructions which go beyond Fox-Milnor. We also refer to KL99b, Ta02, HKL08, Liv09 and also FV09 for more on twisted Alexander polynomials of knots and their relation to knot concordance. 
Remark. (1) If $\alpha$ is the trivial representation, then Theorem 1.1 was proved in the one-variable case by Murasugi Mu67. (cf. also Ka77]) and in the multi-variable case it was proved independently by Kawauchi Ka78, Theorem B] and Nakagawa [Na78. These results were reproved by Turaev Tu86, Theorem 5.4.2] using torsion.

(2) Theorem 1.1 is related in spirit to [Fr05, Theorem 2.2] where a sliceness obstruction for links is given using the Atiyah-Patodi-Singer eta invariant and unitary representations factoring through $p$-groups. The metabelian case was considered earlier by the first author and Ko CKo99.

(3) We expect the obstruction of Theorem 1.1 to be closely related to the discriminant part of Hirzebruch-type invariants developed by the first author [Ch10].

We prove a generalization of Milnor's classical duality theorem for Reidemeister torsion Mi62 and use it as a key ingredient in the proof of the above sliceness obstruction. For an even dimensional compact oriented manifold $W$ endowed with a homomorphism $\mathbb{Z}\left[\pi_{1}(W)\right] \rightarrow Q$ into a field $Q$ with involution such that $W$ is $Q$-acyclic, Milnor proved that the Reidemeister torsion of $M=\partial W$ over $Q$ is of the form $q \bar{q}$, where $q$ is the Reidemeister torsion of $W$. In particular, up to norms, the torsion of $M$ is trivial. A generalization of this to the twisted acyclic case is given in KL99a]. We generalize these prior results to the non-acyclic case: the twisted torsion of an odd dimensional manifold $M$ is equal to the determinant of the twisted intersection pairing of a bounding even-dimensional manifold $W$ (up to norms). For a precise statement, see Theorem 2.4. We hope that this is of independent interest and useful for further applications.

The paper is organized as follows. In Section 2 we introduce twisted torsion of 3-manifolds and links with non-acyclic twisted homology and we show how these invariants relate to intersection forms of bounding 4-manifolds. In Section 3 we study the torsion invariant of homology cobordant 3-manifolds and in Section 4 we apply these results to link concordance where we prove our main theorem (which implies Theorem 1.1). In Section 5 we discuss and to a certain degree classify complex representations which factor through $p$-groups. In Section 6 we show how to compute twisted torsion of boundary links using a boundary link Seifert matrix and we discuss the behavior of our invariants for links which are boundary slice. Finally in Section 7 we prove a formula for the twisted invariants of satellite links and we use this formula to reprove the fact (first proved by the first author [Ch10]) that the Bing double of the Figure 8 knot is not slice.

Conventions. The following are understood unless it says specifically otherwise: (i) groups are finitely generated, (ii) manifolds are compact, orientable and connected, (iii) rings are understood to be associative, commutative with unit element, and (iv) homology and cohomology groups are taken with integral coefficients.

Acknowledgments. We would like to thank Baskar Balasubramanyam, David Cimasoni, Taehee Kim, Vladimir Turaev and Liam Watson for helpful comments and conversations. We also thank an anonymous referee for useful suggestions. The first author was supported by the National Research Foundation of Korea (NRF) grants funded by Korean government(MEST) (Grant No. 2009-0094069 and R012007-000-11687-0).

\section{Twisted TORSION OF 3-MANifOLDS AND LINKS}

In this section we define twisted torsion invariants of odd dimensional manifolds for finite dimensional unitary representations for which the corresponding twisted homology groups are not necessarily acyclic. We furthermore investigate twisted 
torsion of odd dimensional manifolds cobounding a manifold, along the way we generalize a theorem of Milnor to the nonacyclic case. In the last section we then specialize to the case of 3-manifolds and link exteriors.

2.1. Twisted homology and Poincaré duality. Let $(X, Y)$ be a CW-pair with $\pi=\pi_{1}(X)$, and $\phi: \pi \rightarrow \mathrm{GL}(k, R)$ a finite dimensional representation over a $\operatorname{ring} R$. Denote by $p: \widetilde{X} \rightarrow X$ the universal covering of $X$ and write $\widetilde{Y}=p^{-1}(Y)$. Recall that $\pi$ acts on the left of $\widetilde{X}$ as the deck transformation group. We consider the cellular chain complex $C_{*}(\widetilde{X}, \widetilde{Y})$ as a right $\mathbb{Z} \pi$-module via $\sigma \cdot g:=g^{-1} \sigma$ for a chain $\sigma$. The $R^{k}$-coefficient cellular chain complex of $(X, Y)$ is defined to be

$$
C_{*}\left(X, Y ; R^{k}\right)=C_{*}(\widetilde{X}, \widetilde{Y}) \otimes_{\mathbb{Z} \pi} R^{k} .
$$

where $R^{k}$ is viewed as a $(\mathbb{Z} \pi, R)$-bimodule via the representation $\phi$. We define the ith twisted homology group to be the $R$-module

$$
H_{i}^{\phi}\left(X, Y ; R^{k}\right)=H_{i}\left(C_{*}\left(X, Y ; R^{k}\right)\right) .
$$

If the representation is understood clearly, then we just write $H_{i}$ instead of $H_{i}^{\phi}$.

Now assume that $R$ is a ring with a (possibly trivial) involution ${ }^{-}$. Given an $R-$ module $A$ we denote by $\bar{A}$ the opposite $R$-module, i.e. $A=\bar{A}$ as abelian groups, and the multiplication by $r \in R$ on $\bar{A}$ is given by the multiplication by $\bar{r}$ on $A$. Finally recall that a representation $\phi: \pi \rightarrow \mathrm{GL}(k, R)$ is called unitary if $\phi\left(g^{-1}\right)=\overline{\phi(g)}^{t}$ for all $g \in \pi$.

We now have the following twisted Poincaré duality theorem (cf. also Le94, p. 91], [FK06. Lemma 4.12], and [KL99a]). Here, given a vector space $V$ over a field $Q$, we denote the dual vector space by $V^{*}=\operatorname{Hom}_{Q}(V, Q)$.

Theorem 2.1. Let $W$ be an $n$-manifold with $\partial W=M \cup M^{\prime}, M$ and $M^{\prime}$ submanifolds such that $M \cap M^{\prime}=\partial M=\partial M^{\prime}$. Let $\phi: \pi_{1}(W) \rightarrow \operatorname{GL}(k, Q)$ be a unitary representation over a field $Q$ with involution. Then there exists a natural isomorphism

$$
H_{i}\left(W, M ; Q^{k}\right) \cong \overline{H_{n-i}\left(W, M^{\prime} ; Q^{k}\right)^{*}}
$$

of $Q$-vector spaces.

The theorem can be proved along the same lines as the standard proof of Poincaré duality. For the reader's convenience we give an outline of the proof.

Proof. Choose a cell structure of $(W, M)$ to define $C_{*}(\widetilde{W}, \widetilde{M})$ and let $D_{*}\left(\widetilde{W}, \widetilde{M^{\prime}}\right)$ be the chain complex of the dual cell structure. Then the equivariant intersection pairing (e.g., see [Le94, p. 91]) gives an isomorphism of chain complexes

$$
C_{*}\left(W, M ; Q^{k}\right) \cong \overline{D_{n-*}\left(W, M^{\prime} ; Q^{k}\right)^{*}} .
$$

Applying the universal coefficient theorem over $Q$, the conclusion follows.

\subsection{Basic definitions of twisted torsion.}

Torsion of a based chain complex. We begin by recalling the algebraic setup for torsion invariants. Suppose $C=\left\{C_{*}\right\}$ is a based chain complex over a field $Q$ and $\mathcal{B}=\left\{\mathcal{B}_{*}\right\}$ is a basis for $H_{*}(C)$, i.e., $\mathcal{B}_{i}$ is a basis of the $Q$-vector spaces $H_{i}(C)$. The torsion $\tau(C, \mathcal{B}) \in Q^{\times}:=Q \backslash\{0\}$ is defined as in [Tu01, Tu02]. (See also Milnor's classic introduction to torsion [Mi66], but note that we follow Turaev's convention, which gives the reciprocal of the torsion in [Mi66].) If $H_{*}(C)$ is identically zero, then we will just write $\tau(C) \in \mathbb{Q}^{\times}$for the torsion.

In the following theorem, we collect well-known algebraic properties of torsion which will be useful later. In the theorem, given two bases $b$ and $b^{\prime}$ of a vector space, we will denote the basis change matrix from $b$ to $b^{\prime}$ by $\left(b \mid b^{\prime}\right)$, as in Milnor Mi66 and Turaev [Tu01, Tu02]. 
Theorem 2.2. Suppose $C$ is a based chain complex over $Q$ and $\mathcal{B}=\left\{B_{i}\right\}$ is a basis of $H_{*}(C)$.

(1) Suppose $\mathcal{B}^{\prime}=\left\{\mathcal{B}_{i}^{\prime}\right\}$ is another basis of $H_{*}(C)$. Then

$$
\tau(C, \mathcal{B})=\tau\left(C, \mathcal{B}^{\prime}\right) \cdot \prod_{i}\left(\mathcal{B}_{i} \mid \mathcal{B}_{i}^{\prime}\right)^{(-1)^{i+1}}
$$

(2) Let $C^{\prime}$ be the dual based chain complex given by $C_{i}^{\prime}=\left(C_{n-i}\right)^{*}$ and $\mathcal{B}^{\prime}$ be the basis of $H_{*}\left(C^{\prime}\right)=H_{n-*}(C)^{*}$ dual to $\mathcal{B}$. Then $\tau(C, \mathcal{B})=\tau\left(C^{\prime}, \mathcal{B}^{\prime}\right)^{(-1)^{n+1}}$.

(3) Suppose $C_{*}$ is acyclic. Choose a basis of the nth cycle submodule $Z_{n}=$ $\operatorname{Ker}\left\{C_{n} \rightarrow C_{n-1}\right\}$, and view

$$
\begin{aligned}
C^{\prime} & =\left\{Z_{n} \longrightarrow C_{n} \longrightarrow \cdots \longrightarrow C_{0}\right\} \\
C^{\prime \prime} & =\left\{\cdots \longrightarrow C_{n+2} \longrightarrow C_{n+1} \longrightarrow Z_{n}\right\}
\end{aligned}
$$

as acyclic based chain complexes (indexed so that $C_{0}^{\prime}=C_{0}, C_{0}^{\prime \prime}=Z_{n}$ ). Then $\tau(C)=\tau\left(C^{\prime}\right) \cdot \tau\left(C^{\prime \prime}\right)^{(-1)^{n}}$.

(4) Suppose $0 \rightarrow C^{\prime} \rightarrow C \rightarrow C^{\prime \prime} \rightarrow 0$ is a short exact sequence of based chain complexes and $\mathcal{B}^{\prime}, \mathcal{B}$, and $\mathcal{B}^{\prime \prime}$ are bases of $H_{*}\left(C^{\prime}\right), H_{*}(C)$ and $H_{*}\left(C^{\prime \prime}\right)$, respectively. We view the associated homology long exact sequence as an acyclic complex, say $H$, based by $\mathcal{B}, \mathcal{B}^{\prime}, \mathcal{B}^{\prime \prime}$. Then

$$
\tau(C, \mathcal{B})=\tau\left(C^{\prime}, \mathcal{B}^{\prime}\right) \cdot \tau\left(C^{\prime \prime}, \mathcal{B}^{\prime \prime}\right) \cdot \tau(H)
$$

Twisted torsion of $C W$-complexes. Let $(X, Y)$ be a finite CW-pair with $\pi=\pi_{1}(X)$ and let $\phi: \pi \rightarrow \operatorname{GL}(k, Q)$ be a representation over a field $Q$. Let $\mathcal{B}=\left\{\mathcal{B}_{*}\right\}$ be a basis of $H_{*}\left(X, Y ; Q^{k}\right)$. The universal cover $(\tilde{X}, \tilde{Y})$ has a natural cell structure, and the chain complex $C_{*}(\tilde{X}, \tilde{Y})$ can be based over $\mathbb{Z} \pi$ by choosing a lift of each cell of $(X, Y)$ and orienting it. This, together with the standard basis of $Q^{k}$, gives rise to a basing of $C_{*}\left(X, Y ; Q^{k}\right)$ over $Q$. We can then define the twisted torsion

$$
\tau^{\phi}(X, Y, \mathcal{B}) \in Q^{\times}
$$

to be the torsion of $C_{*}\left(X, Y ; Q^{k}\right)$ with respect to $\mathcal{B}$. We will drop $\mathcal{B}$ from the notation if $H_{*}\left(X, Y ; Q^{k}\right)=0$.

Standard arguments (e.g., see [Mi66, Tu86, Tu01, Tu02]) show that $\tau^{\phi}(X, Y, \mathcal{B})$ is well-defined up to multiplication by an element in $\pm \operatorname{det}(\phi(\pi))$, and is invariant under simple homotopy preserving $\mathcal{B}$. By Chapman's theorem Chp74 the invariant $\tau^{\phi}(X, Y, \mathcal{B})$ only depends on the homeomorphism type of $(X, Y)$. In particular when $(M, N)$ is a manifold pair, we can define $\tau^{\phi}(M, N, \mathcal{B})$ by picking any finite CW-structure for $(M, N)$.

Twisted torsion of odd dimensional manifolds. Let $M$ be an odd dimensional manifold with $\pi=\pi_{1}(M)$ and let $\phi: \pi \rightarrow \operatorname{GL}(k, Q)$ be a representation over a field $Q$. In many interesting cases $H_{*}\left(M ; Q^{k}\right)$ will be nontrivial. For example the untwisted multivariable Alexander module of a boundary link with at least two components is always non-torsion. In order to define an invariant for the nonacyclic case without referring to a basis of homology, we now assume the following two conditions hold:

(1) $Q$ is endowed with a (possibly trivial) involution, and $\phi$ is unitary.

(2) $\partial M$ is $Q^{k}$-acyclic, i.e., $H_{*}\left(\partial M ; Q^{k}\right)=0$.

Since $\phi$ is unitary, we have the Poincaré duality isomorphism

$$
H_{i}\left(M ; Q^{k}\right) \cong \overline{H_{n-i}\left(M, \partial M ; Q^{k}\right)^{*}} \cong \overline{H_{n-i}\left(M ; Q^{k}\right)^{*}}
$$

of $Q$-vector spaces, by Theorem 2.1 and by Condition (2). Since $M$ is odd dimensional, one can pick a basis $\mathcal{B}=\left\{\mathcal{B}_{*}\right\}$ for $H_{*}\left(M ; Q^{k}\right)$ with the following property: for each $i, \mathcal{B}_{i}$ is the dual basis of $\mathcal{B}_{n-i}$ via the above Poincaré duality isomorphism. We call such a basis $\mathcal{B}=\left\{B_{*}\right\}$ a self-dual basis for $H_{*}\left(M ; Q^{k}\right)$. 
Lemma 2.3. Suppose $\mathcal{B}$ and $\mathcal{B}^{\prime}$ are self-dual bases for $H_{*}\left(M ; Q^{k}\right)$. Then for some $q \in Q^{\times}$, up to the indeterminacy of the torsion,

$$
\tau^{\phi}\left(M, \mathcal{B}^{\prime}\right)=\tau^{\phi}(M, \mathcal{B}) \cdot q \bar{q} .
$$

Proof. Let $\left(\mathcal{B}_{i} \mid \mathcal{B}_{i}^{\prime}\right)$ be the determinant of the base change matrix from $\mathcal{B}_{i}$ to $\mathcal{B}_{i}^{\prime}$. Then $\left(\mathcal{B}_{n-i} \mid \mathcal{B}_{n-i}^{\prime}\right)={\overline{\left(\mathcal{B}_{i} \mid \mathcal{B}_{i}^{\prime}\right)}}^{-1}$. The desired conclusion follows immediately from Theorem 2.2.

We define the norm subgroup of $Q^{\times}$to be $N(Q)=\left\{ \pm q \bar{q} \mid q \in Q^{\times}\right\}$, and we say $f \in Q^{\times}$is a norm when $f \in N(Q)$. We define

$$
\tau^{\phi}(M)=\tau^{\phi}(M, \mathcal{B}) \text { as an element in } Q^{\times} / N(Q)
$$

where $\mathcal{B}$ is a self-dual basis of $H_{*}\left(M ; Q^{k}\right)$. By Lemma 2.3 the invariant $\tau^{\phi}(M)$ is well-defined up to multiplication by an element in $\pm \operatorname{det}\left(\phi\left(\pi_{1}(M)\right)\right)$. This invariant was first introduced by Turaev Tu86. Section 5.1] in the untwisted case.

In the following, given $f \in Q$, we will sometimes write $\tau^{\phi}(M) \doteq f \in Q^{\times} / N(Q)$, to indicate that there exists a representative of $\tau^{\phi}(M)$ which equals $f$.

2.3. Twisted torsion and bounding manifolds. In this subsection we prove a non-acyclic generalization of a well-known theorem of Milnor Mi62, Theorem 2] and of its twisted analogue due to Kirk and Livingston KL99a, Theorem 5.1 and Corollary 5.3].

Let $W$ be a $2 r$-dimensional manifold with (possibly disconnected) boundary $M$. Let $\phi: \pi_{1}(W) \rightarrow \mathrm{GL}(k, Q)$ be a unitary representation. Now consider the map

$$
H_{r}\left(W ; Q^{k}\right) \stackrel{\cong}{\longrightarrow} \overline{H_{r}\left(W, M^{\prime} ; Q^{k}\right)^{*}} \stackrel{\iota^{*}}{\longrightarrow} \overline{H_{r}\left(W ; Q^{k}\right)^{*}}
$$

where the first map is the isomorphism given by Theorem 2.1 and the second map is induced by $\iota:(W, \emptyset) \rightarrow(W, M)$. This map gives rise to a pairing

$$
\lambda: H_{r}\left(W ; Q^{k}\right) \longrightarrow H_{r}\left(W ; Q^{k}\right) \longrightarrow Q
$$

which is well-known to be $(-1)^{r}$-hermitian. This pairing is called the equivariant intersection form of $(W, \phi)$. This form is in general singular, in fact for any $x \in$ $\operatorname{Im}\left\{H_{r}\left(M ; Q^{k}\right) \rightarrow H_{r}\left(W ; Q^{k}\right)\right\}$ and $y \in H_{r}\left(W ; Q^{k}\right)$ we have $\lambda(x, y)=\lambda(y, x)=0$. In particular $\lambda$ gives rise to a pairing on $H_{r}\left(W ; Q^{k}\right) / i_{*} H_{r}\left(M ; Q^{k}\right)$ which turns out to be non-singular.

We now pick a basis $\mathcal{B}=\left\{v_{1}, \ldots, v_{s}\right\}$ for $H_{r}\left(W ; Q^{k}\right) / i_{*} H_{r}\left(M ; Q^{k}\right)$ and we compute $\operatorname{det}\left(\lambda\left(v_{i}, v_{j}\right)\right) \in Q^{\times}$. Note that if we change the basis, then the determinant of the form changes by a norm. Put differently, we obtain a well-defined invariant

$$
\Lambda(W, \phi):=\operatorname{det}\left(\lambda\left(v_{i}, v_{j}\right)\right) \in Q^{\times} / N(Q) .
$$

Note that $\Lambda(W, \phi)=\overline{\Lambda(W, \phi)}$ since $\lambda$ is $(-1)^{r}$-hermitian.

Theorem 2.4. Suppose $W$ is a $2 r$-dimensional manifold with (possibly disconnected) boundary $M$. Let $\phi: \pi_{1}(W) \rightarrow \mathrm{GL}(k, Q)$ be a unitary representation. Then

$$
\tau^{\phi}(M)=\Lambda(W, \phi) \in Q^{\times} / N(Q)
$$

up to the indeterminacy $\pm \operatorname{det}\left(\phi\left(\pi_{1}(M)\right)\right)$.

In the following we will only apply the theorem to the case that $\Lambda(W, \phi)=1$, but we hope that the general case is of independent interest.

To prove Theorem 2.4 we need the following duality of torsion, which is essentially due to Milnor Mi62. (See also Kirk and Livingston KL99a for the twisted case.) 
Lemma 2.5. Suppose $W$ is an n-manifold and $M, M^{\prime}$ are submanifolds of $\partial W$ such that $\partial W=M \cup M^{\prime}$ and $M \cap M^{\prime}=\partial M=\partial M^{\prime}$. Suppose $\phi: \pi_{1}(W) \rightarrow \operatorname{GL}(k, Q)$ is a unitary representation, $\mathcal{B}=\left\{\mathcal{B}_{*}\right\}$ is a basis for $H_{*}\left(W, M ; Q^{k}\right)$, and $\mathcal{B}^{\prime}$ is the dual basis for $H_{*}\left(W, M^{\prime} ; Q^{k}\right)=\overline{H_{n-*}\left(W, M ; Q^{k}\right)^{*}}$. Then

$$
\tau^{\phi}(W, M, \mathcal{B})=\overline{\tau^{\phi}\left(W, M^{\prime}, \mathcal{B}^{\prime}\right)}(-1)^{n+1}
$$

Proof. The lemma follows immediately from the duality

$$
C_{i}\left(W, M ; Q^{k}\right) \cong \overline{D_{n-i}\left(W, M^{\prime} ; Q^{k}\right)^{*}}
$$

(see the proof of Theorem 2.1) and Theorem 2.2.

Proof of Theorem 2.4. Choose a basis $\mathcal{B}$ for $H_{*}\left(W ; Q^{k}\right)$, and choose a self-dual basis $\mathcal{B}^{\prime}$ for $H_{*}\left(M ; Q^{k}\right)$. Let $\mathcal{B}^{\prime \prime}$ be the basis of $H_{*}\left(W, M ; Q^{k}\right)=\overline{H_{n-*}\left(W ; Q^{k}\right)^{*}}$ which is dual to $\mathcal{B}$. From (the proof of) Theorem 2.1. Theorem 2.2. and Lemma 2.5. it follows that

$$
\tau^{\phi}(W, \mathcal{B})=\tau_{0} \cdot \tau^{\phi}\left(M, \mathcal{B}^{\prime}\right) \cdot{\overline{\tau^{\phi}(W, \mathcal{B})}}^{(-1)^{n+1}}
$$

where $\tau_{0}$ is the torsion of the acyclic chain complex (= homology long exact sequence)

$\cdots \longrightarrow H_{i+1}\left(W, M ; Q^{k}\right) \longrightarrow H_{i}\left(M ; Q^{k}\right) \longrightarrow H_{i}\left(W ; Q^{k}\right) \longrightarrow H_{i}\left(W, M ; Q^{k}\right) \longrightarrow \cdots$

which is based by $\mathcal{B}, \mathcal{B}^{\prime}, \mathcal{B}^{\prime \prime}$. We break the long exact sequence at $i_{*}: H_{r}\left(W ; Q^{k}\right) \rightarrow$ $H_{r}\left(W, M ; Q^{k}\right)$ as in Theorem 2.2, let $P$ be the image of $i_{*}$, choose a basis $b$ for $P$, and let

$$
\begin{aligned}
C^{\prime} & =\left\{P \longrightarrow H_{r}\left(W, M ; Q^{k}\right) \longrightarrow \cdots \longrightarrow H_{0}\left(W, M ; Q^{k}\right)\right\}, \\
C^{\prime \prime} & =\left\{\cdots \longrightarrow H_{r}\left(M ; Q^{k}\right) \longrightarrow H_{r}\left(W ; Q^{k}\right) \longrightarrow P\right\} .
\end{aligned}
$$

Then by Theorem 2.2 (3) we have $\tau_{0}=\tau\left(C^{\prime}\right) \tau\left(C^{\prime \prime}\right)^{(-1)^{r}}$. Since $\mathcal{B}^{\prime}$ is self-dual, $C^{\prime \prime}=\left\{C_{i}^{\prime \prime}\right\}$ is canonically isomorphic, as a based chain complex, with the dual chain complex $\left\{{\overline{C_{3 r+1-i}^{\prime}}}^{*}\right\}$ of $C^{\prime}$ except $C_{0}^{\prime \prime}=P$. Therefore we have

$$
\tau\left(C^{\prime \prime}\right)={\overline{\tau\left(C^{\prime}\right)}}^{(-1)^{r}} \cdot\left(\overline{b^{*}} \mid b\right)
$$

where $b^{*}$ is the dual basis of $b$ for $P^{*}$. It follows that

$$
\tau^{\phi}\left(M, \mathcal{B}^{\prime}\right)=\tau^{\phi}(W, \mathcal{B}) \cdot \overline{\tau^{\phi}(W, \mathcal{B})} \cdot \tau\left(C^{\prime}\right)^{-1} \cdot \overline{\tau\left(C^{\prime}\right)}-1 \cdot\left(b \mid \overline{b^{*}}\right)^{(-1)^{r}} .
$$

Note that by definition we have $\left(b \mid \overline{b^{*}}\right)=\Lambda(W, \phi)=: D \in Q^{\times} / N(Q)$. Since the intersection form is $(-1)^{r}$-hermitian, $D= \pm \bar{D}$ and so $D^{-1} \equiv D$ in $Q^{\times} / N(Q)$. This completes the proof.

Remark. If $W$ is $Q^{k}$-acyclic, then $D=1$ automatically and $\tau\left(C^{\prime}\right)=1$ in the above proof. So $\tau^{\phi}(M)=q \bar{q}$ up to $\pm \operatorname{det}\left(\phi\left(\pi_{1}(M)\right)\right)$, where $q=\tau^{\phi}(W)$. This shows our non-acyclic result specializes to Milnor's theorem [Mi62, Theorem 2] and its twisted analogue due to Kirk and Livingston [KL99a, Theorem 5.1 and Corollary 5.3].

2.4. Twisted torsion of 3-manifolds and links. In this section we now specialize to the case of 3-manifolds. Let $M$ be a 3-manifold with empty or toroidal boundary (e.g. a link exterior). We write $\pi=\pi_{1}(M)$. Let $\psi: \pi \rightarrow H$ be an epimorphism onto a nontrivial free abelian group $H$. Let $R$ be a domain with involution. We equip $R[H]$ with the involution given by $\overline{r h}=\bar{r} h^{-1}$ for $r \in R$ and $h \in H$. This extends to an involution on $Q(H)$, the quotient field of $R[H]$. Now let $\alpha: \pi \rightarrow \operatorname{GL}(k, R)$ be a unitary representation. Using $\alpha$ and $\psi$, we define a left $\mathbb{Z}[\pi]$-module structure on $R[H]^{k}:=R^{k} \otimes_{R} R[H]$ as follows:

$$
g \cdot(v \otimes p):=(\alpha(g) \cdot v) \otimes(\psi(g) p)
$$


where $g \in \pi, v \in R^{k}$ and $p \in R[H]$. This extends to a $\mathbb{Z}[\pi]$-module structure on $Q(H)^{k}$. It can be seen that $\alpha \otimes \psi: \pi \rightarrow \mathrm{GL}(k, Q(H))$ is unitary since $\alpha$ is unitary. We will several times make use of the following simple fact:

$$
\operatorname{det}((\alpha \otimes \psi)(\pi)) \subset \operatorname{det}(\alpha(\pi)) \cdot H .
$$

We now say that $\psi: \pi \rightarrow H$ is admissible if $\psi$ restricted to any boundary component of $M$ is nontrivial. Note that $\psi$ is always admissible if $M$ is closed. If $\psi$ is admissible then Condition (2) in Section 2.2 is satisfied (e.g., see [KL99a, Proposition 3.5] and KL99a, Section 3.3]) and by the discussion of the previous section we thus obtain an invariant

$$
\tau^{\alpha \otimes \psi}(M) \in Q(H)^{\times} / N(Q(H))
$$

well-defined up to multiplication by an element of the form $\pm d h$ with $d \in \operatorname{det}(\alpha(\pi))$ and $h \in H$.

Remark. Note that if $\operatorname{rank}(H)$ is nonzero, then in the above setting we have $H_{i}\left(M ; Q(H)^{k}\right)=0$ for $i=0,3$ (see e.g. [FK06, FK08]), so that it suffices to choose $\mathcal{B}_{1}$ in the definition of torsion.

We now specialize even further, namely to the case of link exteriors. Let $L \subset S^{3}$ be an oriented $m$-component link. We denote the exterior by $E_{L}$ and the (oriented) meridians by $\mu_{1}, \ldots, \mu_{m}$. Using the basis $\mu_{1}, \ldots, \mu_{m}$ we can now naturally identify $H_{1}\left(E_{L} ; \mathbb{Z}\right)$ with $\mathbb{Z}^{m}$. We say that a homomorphism $\psi: \mathbb{Z}^{m} \rightarrow H$ is admissible if $\psi$ is an epimorphism onto a nontrivial free abelian group $H$ such that the epimorphism is nontrivial on each subsummand of $\mathbb{Z}^{m}=\mathbb{Z} \oplus \cdots \oplus \mathbb{Z}$. In this case the induced map $\pi_{1}\left(E_{L}\right) \rightarrow H$ is admissible in the above sense, so that we obtain a well-defined torsion invariant

$$
\tau^{\alpha \otimes \psi}(L):=\tau^{\alpha \otimes \psi}\left(E_{L}\right) \in Q(H)^{\times} / N(Q(H))
$$

\section{HoMOlOGY COBORDISM AND TWISTED TORSION}

In this section we investigate the behaviour of the twisted torsion under homology cobordism. Recall that two 3-manifolds $M$ and $M^{\prime}$ (possibly with nonempty toroidal boundary) are said to be homology cobordant if there exists a 4 -manifold $W$ containing $M$ and $M^{\prime}$ as submanifolds such that $\partial W=M \cup-M^{\prime}, \partial M=M \cap M^{\prime}=$ $\partial M^{\prime}$, and the inclusions $M \rightarrow W$ and $M^{\prime} \rightarrow W$ induce isomorphisms on $H_{*}(-; \mathbb{Z})$.

From now on, $R \subset \mathbb{C}$ is always assumed to be a subring closed under complex conjugation. We denote by $P_{k}(R, \pi)$ the set of representations $\pi \rightarrow \operatorname{GL}(k, R)$ factoring through a $p$-group. We will continuously make use of the well-known fact that complex representations factoring through finite groups are necessarily unitary.

For $\psi: \pi_{1}(M) \rightarrow H$ and $\alpha \in P_{k}(R, \pi)$, we define

$$
\operatorname{rank}(M, \alpha, \psi):=\operatorname{dim}_{Q(H)} H_{1}^{\alpha \otimes \psi}\left(M ; Q(H)^{k}\right) .
$$

Our main result is the following:

Theorem 3.1. Suppose two 3-manifolds $M$ and $M^{\prime}$ are homology cobordant and $H$ is a free abelian group. Then there are bijections

$$
\begin{gathered}
\Psi: \operatorname{Hom}\left(\pi_{1}(M), H\right) \longrightarrow \operatorname{Hom}\left(\pi_{1}\left(M^{\prime}\right), H\right) \\
\Phi: P_{k}\left(R, \pi_{1}(M)\right) \longrightarrow P_{k}\left(R, \pi_{1}\left(M^{\prime}\right)\right)
\end{gathered}
$$

such that for any $\psi: \pi_{1}(M) \rightarrow H, \psi$ is admissible if and only if $\Psi(\psi)$ is admissible, and in this case, for any $\alpha \in P_{k}\left(R, \pi_{1}(M)\right)$, we have

$$
\operatorname{rank}(M, \alpha, \psi)=\operatorname{rank}\left(M^{\prime}, \Phi(\alpha), \Psi(\psi)\right)
$$


and

$$
\tau^{\alpha \otimes \psi}(M)= \pm d h \tau^{\Phi(\alpha) \otimes \Psi(\psi)}\left(M^{\prime}\right) \text { in } Q(H)^{\times} / N(Q(H))
$$

for some $d \in \operatorname{det}\left(\alpha\left(\pi_{1}(M)\right)\right)=\operatorname{det}\left((\Psi(\alpha))\left(\pi_{1}\left(M^{\prime}\right)\right)\right)$ and $h \in H$.

Remark. In the proof of Theorem 3.1 we will see that for a given homology cobor$\operatorname{dism} W$ between $M$ and $M^{\prime}$, the maps $\Psi$ and $\Phi$ are both induced by $\pi_{1}(M) \rightarrow$ $\pi_{1}(W) \leftarrow \pi_{1}\left(M^{\prime}\right)$, in particular $\Psi$ and $\Phi$ are 'related' bijections.

3.1. Stallings Theorem and representations through $p$-groups. We first construct the bijections in Theorem 3.1] using Stallings' theorem Sta65.

Proof of Theorem 3.1, Part I. Suppose $W$ is a homology cobordism between $M$ and $M^{\prime}$. Obviously the induced isomorphisms on $H_{1}(-; \mathbb{Z})$ gives rise to bijections

$$
\operatorname{Hom}\left(\pi_{1}(M), H\right) \approx \operatorname{Hom}\left(\pi_{1}(W), H\right) \approx \operatorname{Hom}\left(\pi_{1}\left(M^{\prime}\right), H\right) .
$$

Their composition will be $\Psi$ in the above statement. Since $\partial M=\partial M^{\prime}$ it follows that $\psi: \pi_{1}(M) \rightarrow H$ is admissible if and only if so is $\Psi(\psi)$.

The bijection $\Phi$ is constructed as follows. For a group $G$, we denote the $q$ th lower central subgroup by $G_{q}$, which is defined inductively via $G_{1}=G, G_{q}=\left[G, G_{q-1}\right]$. For any $\alpha \in P_{k}\left(R, \pi_{1}(M)\right), \alpha$ factors through $\pi_{1}(M) / \pi_{1}(M)_{q}$ for some $q$, since any $p$-group is nilpotent. By Stallings' theorem [Sta65], we have

$$
\pi_{1}(M) / \pi_{1}(M)_{q} \stackrel{\cong}{\longrightarrow} \pi_{1}(W) / \pi_{1}(W)_{q} .
$$

Therefore $\alpha$ induces a representation $\pi_{1}(W) \rightarrow \mathrm{GL}(k, R)$ which factors through $\pi_{1}(W) / \pi_{1}(W)_{q}$. It is easily seen that this induces a bijection $P_{k}\left(R, \pi_{1}(M)\right) \approx$ $P_{k}\left(R, \pi_{1}(W)\right)$. Similarly for $M^{\prime}$ and $W$, and composing them, we obtain a bijection $\Phi: P_{k}\left(R, \pi_{1}(M)\right) \rightarrow P_{k}\left(R, \pi_{1}\left(M^{\prime}\right)\right)$.

3.2. Cohn local property and twisted coefficients. In our proof of the conclusion on torsions in Theorem 3.1 we will compute (the quotient of) the torsions of $M$ and $M^{\prime}$ in terms of the $Q(H)^{k}$-coefficient intersection pairing of a cobordism $W$, appealing to Theorem 2.4. The key point is that one can prove that the intersection pairing is trivial when $W$ is a homology cobordism. This is done following a standard strategy, namely by controlling the size of the underlying $Q(H)^{k}$-coefficient homology of $(W, M)$, appealing to a chain contraction argument originally due to Vogel.

In Lemma 3.2 below we denote by $\epsilon: \mathbb{Z}[\pi] \rightarrow \mathbb{Z}$ the augmentation map given by $g \mapsto 1, g \in \pi$.

Lemma 3.2. Suppose $\pi$ is a group and $R$ is a domain with characteristic zero, $\alpha \in P_{k}(R, \pi)$, and $\psi: \pi \rightarrow H$ is an epimorphism onto a nontrivial free abelian group $H$. If $A$ is a square matrix over $\mathbb{Z}[\pi]$ such that $\epsilon(A)$ is invertible over $\mathbb{Z}$, then $(\alpha \otimes \psi)(A)$ is invertible over $Q(H)$.

Remark. Another way of phrasing Lemma 3.2 is to say that the $\mathbb{Z}[\pi]$-module $Q(H)^{k}$ is in fact a module over the Cohn localization of $\mathbb{Z}[\pi]$.

Our proof of Lemma 3.2 depends heavily on a result originally due to Strebel Str74] and Levine Le94.

Proof. Since $R$ has characteristic zero and since the quotient ring of $R$ gives the same $Q(H)$, we may assume that $R$ contains $\mathbb{Q}$. Suppose $\alpha$ factors through $f: \pi \rightarrow$ 
$P$ where $P$ is a $p$-group. We have the following commutative diagram:

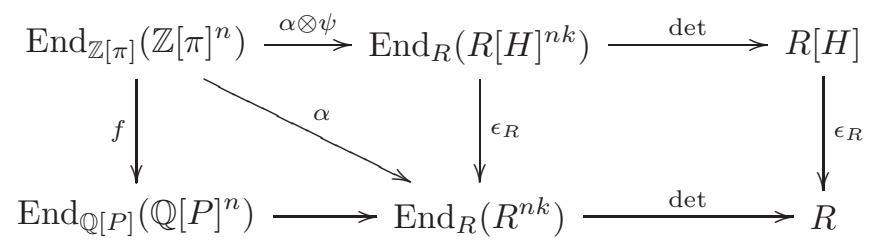

where the induced maps are denoted by the same symbol, as an abuse of notation. The augmentation $R[H] \rightarrow R$ is denoted by $\epsilon_{R}$.

Suppose $A \in \operatorname{End}_{\mathbb{Z}[\pi]}\left(\mathbb{Z}[\pi]^{n}\right)$ is an $n \times n$ matrix over $\mathbb{Z}[\pi]$ such that $\epsilon(A)$ is invertible. Due to Strebel [Str74, Lemma 1.10] and Levine Le94, Lemma 4.3] this implies that $f(A)$ is invertible over $\mathbb{Q}[P]$, i.e., a unit in $\operatorname{End}_{\mathbb{Q}[P]}\left(\mathbb{Q}[P]^{n}\right)$. Since the maps in the above diagram are multiplicative, it follows that $\alpha(A)$ is invertible. Therefore $\operatorname{det}(\alpha(A)) \neq 0$ and $\operatorname{det}((\alpha \otimes \psi)(A)) \neq 0$. It follows that $A$ is injective over $R[H]$ and hence invertible over the field $Q(H)$.

A standard well-known chain contraction argument originally due to Vogel (see also Levine [Le94, Proposition 4.2] and Cochran-Orr-Teichner [COT03]) shows the following statement as a consequence of Lemma 3.2 .

Lemma 3.3. Suppose $(X, A)$ is a finite $C W$-pair, $R$ is of characteristic zero, $\alpha \in$ $P_{k}\left(R, \pi_{1}(X)\right)$, and $\psi: \pi_{1}(X) \rightarrow H$ is an epimorphism onto a nontrivial free abelian group. If $H_{*}(X, A ; \mathbb{Z})=0$, then $H_{*}\left(X, A ; Q(H)^{k}\right)=0$.

Proof of Theorem 3.1, Part II. Suppose $W$ is a homology cobordism between $M$ and $M^{\prime}$. Recall that we have constructed bijections

$$
\begin{gathered}
P_{k}\left(R, \pi_{1}(M)\right) \approx P_{k}\left(R, \pi_{1}(W)\right) \approx P_{k}\left(R, \pi_{1}\left(M^{\prime}\right)\right), \\
\operatorname{Hom}\left(\pi_{1}(M), H\right) \approx \operatorname{Hom}\left(\pi_{1}(W), H\right) \approx \operatorname{Hom}\left(\pi_{1}\left(M^{\prime}\right), H\right) .
\end{gathered}
$$

Fix $\alpha \in P_{k}\left(R, \pi_{1}(M)\right)$ and $\psi: \pi_{1}(M) \rightarrow H$, and as an abuse of notation, we denote by $\alpha$ and $\psi$ the corresponding representations and homomorphisms of $\pi_{1}\left(M^{\prime}\right)$ and $\pi_{1}(W)$ as well.

For convenience write $Q=Q(H)$. Since $H_{*}(W, M)=0$, we have $H_{*}\left(W, M ; Q^{k}\right)=$ 0 by Lemma 3.3, and so $H_{*}\left(M ; Q^{k}\right) \cong H_{*}\left(W ; Q^{k}\right)$. Similarly for $M^{\prime}$ and $W$, and from this the conclusion on the ranks follows immediately.

Now pick a self-dual basis $\mathcal{B}$ for $H_{*}\left(M ; Q^{k}\right)$ and pick a self-dual basis $\mathcal{B}^{\prime}$ for $H_{*}\left(M^{\prime} ; Q^{k}\right)$. Recall that $H_{*}\left(\partial M ; Q^{k}\right)=H_{*}\left(\partial M^{\prime} ; Q^{k}\right)=0$ since $\psi$ is admissible (see Section 2.4). In particular $\mathcal{B}$ gives rise to a basis for $H_{*}\left(M, \partial M ; Q^{k}\right)$ which we also denote by $\mathcal{B}$. Note that $\mathcal{B}$ and $\mathcal{B}^{\prime}$ give rise to a self-dual basis for $H_{*}\left(\partial W ; Q^{k}\right)$ which we denote by $\mathcal{B} \oplus \mathcal{B}^{\prime}$.

Since $H_{*}\left(W, M ; Q^{k}\right)=0$, we have $\Lambda(W, \phi)=1$. Therefore, by Theorem 2.4, we have $\tau^{\alpha \otimes \psi}(\partial W) \doteq \tau^{\alpha \otimes \psi}\left(\partial W, \mathcal{B} \oplus \mathcal{B}^{\prime}\right) \doteq 1$. Applying Theorem 2.2 to the excision short exact sequence

$$
0 \longrightarrow C_{*}\left(M^{\prime} ; Q^{k}\right) \longrightarrow C_{*}\left(\partial W ; Q^{k}\right) \longrightarrow C_{*}\left(M, \partial M ; Q^{k}\right) \longrightarrow 0
$$

and then applying Lemma 2.5 we obtain

$$
\begin{aligned}
1=\tau^{\alpha \otimes \psi}\left(\partial W, \mathcal{B} \oplus \mathcal{B}^{\prime}\right) & =\tau^{\alpha \otimes \psi}\left(M^{\prime}, \mathcal{B}^{\prime}\right) \cdot \tau^{\alpha \otimes \psi}(M, \partial M, \mathcal{B}) \cdot \tau_{0} \\
& =\tau^{\alpha \otimes \psi}\left(M^{\prime}, \mathcal{B}^{\prime}\right) \cdot \overline{\tau^{\alpha \otimes \psi}(M, \mathcal{B})} \cdot \tau_{0}
\end{aligned}
$$

where $\tau_{0}$ is the torsion of the homology long exact sequence

$$
\longrightarrow H_{i}\left(M^{\prime} ; Q^{k}\right) \longrightarrow H_{i}\left(\partial W ; Q^{k}\right) \longrightarrow H_{i}\left(M, \partial M ; Q^{k}\right) \longrightarrow H_{i-1}\left(M^{\prime} ; Q^{k}\right) \longrightarrow
$$

which is based by $\mathcal{B}^{\prime}, \mathcal{B}^{\prime} \oplus \mathcal{B}$ and $\mathcal{B}$. It follows easily that $\tau_{0}=1$. Therefore, multiplying $(*)$ by $\tau^{\alpha \otimes \psi}(M, \mathcal{B})$, the conclusion follows. 


\section{LINK CONCORDANCE AND TWISTED TORSION}

It is well known that if two links are concordant, then their exteriors are homology cobordant. More precisely, if $m$-component links $L$ and $L^{\prime}$ in $S^{3}$ are concordant, then the exterior $W$ of a concordance has boundary $E_{L} \cup m\left(S^{1} \times S^{1} \times[0,1]\right) \cup-E_{L^{\prime}}$ where we glue the $i$-th meridian (longitude) of $L$ to the $i$-th meridian (longitude) of $L^{\prime}$ (Recall that links are assumed to be ordered collections of circles). Rounding corners and extending collars outward, we can assume that $\partial W=E_{L} \cup-E_{L^{\prime}}$ and $\partial E_{L}=E_{L} \cap E_{L^{\prime}}=\partial E_{L^{\prime}}$. Using Alexander duality it is straightforward to verify that $W$ is indeed a homology cobordism.

Therefore, we can apply the invariance of torsion (Theorem 3.1) immediately. Furthermore, in case of links, we can make further observations on the correspondence between representations. The main aim of this section is to provide a precise description of this correspondence.

First, recall that given an oriented link $L \subset S^{3}$ with $m$ components, we can naturally identify $H_{1}\left(E_{L}\right)$ with $\mathbb{Z}^{m}$ in such a way that the $i$ th positive meridian $\mu_{i}$ of $L$ represents the $i$ th standard basis of $\mathbb{Z}^{m}$. Therefore $\operatorname{Hom}\left(\pi_{1}\left(E_{L}\right), H\right)$ is identified with $\operatorname{Hom}\left(\mathbb{Z}^{m}, H\right)$, where $H$ denotes a nontrivial free abelian group as in the previous sections. Suppose two links $L$ and $L^{\prime}$ are concordant, in particular the concordance exterior $W$ is a homology cobordism between $E_{L}$ and $E_{L^{\prime}}$. Recall that in Theorem 3.1, we defined a bijection $\Psi: \operatorname{Hom}\left(\pi_{1}\left(E_{L}\right), H\right) \rightarrow \operatorname{Hom}\left(\pi_{1}\left(E_{L^{\prime}}\right), H\right)$ using $H_{1}\left(E_{L}\right) \cong H_{1}(W) \cong H_{1}\left(E_{L^{\prime}}\right)$. Since the meridians of $L$ and $L^{\prime}$ are freely homotopic in $W$, it is easily seen that the bijection $\Psi$ induces the identity on $\operatorname{Hom}\left(\mathbb{Z}^{m}, H\right)$, that is, a homomorphisms $\pi_{1}\left(E_{L}\right) \rightarrow H$ and its image under $\Psi$ are identified with the same homomorphism $\mathbb{Z}^{m} \rightarrow H$.

Recall that a homomorphism $\psi: \mathbb{Z}^{m} \rightarrow H$ is admissible if $\psi$ is an epimorphism which is nontrivial on each $\mu_{i}$. In this case the corresponding map $\pi_{1}\left(E_{L}\right) \rightarrow H$, which will also be denoted by $\phi$, is admissible in the sense of Section 2 . Thus the twisted torsion $\tau^{\alpha \otimes \phi}\left(E_{L}\right)$ can be defined for any unitary representation $\alpha: \pi_{1}\left(E_{L}\right) \rightarrow$ $\mathrm{GL}(k, R)$.

The correspondence between representations of $\pi=\pi_{1}(-)$ for concordant links is better described in terms of representations of the lower central quotients $\pi / \pi_{q}$. It causes no loss of generality, since any representation of $\pi$ factoring through a $p$-group factors through $\pi / \pi_{q}$ for some $q$ since $p$-groups are nilpotent. To proceed we will need some technicalities discussed in the following subsection.

4.1. Concordance and lower central series. In this subsection we state some known results and some folklore results on link concordance and lower central series.

Let $L \subset S^{3}$ be an ordered, oriented $m$-component link. We denote the meridians by $\mu_{1}, \ldots, \mu_{m}$ and by abuse of notation we will denote the corresponding elements in $\pi_{1}\left(E_{L}\right)$ by $\mu_{1}, \ldots, \mu_{m}$ as well. We now denote by $F$ the free group on $m$ generators $x_{1}, \ldots, x_{m}$. Following ideas of Levine (see [Le94, p. 101]) we define an $F / F_{q^{-}}$ structure for $L$ to be a homomorphism $\varphi: \pi \rightarrow F / F_{q}$ such that for any $i=1, \ldots, m$ the element $\varphi\left(\mu_{i}\right)$ is a conjugate of $x_{i}$. (Here recall that given a group $\pi$ we denote by $\pi_{q}$ the $q$ th lower central subgroup.) Furthermore, we refer to a link $L$ equipped with an $F / F_{q}$-structure as an $F / F_{q}$-link.

A special automorphism of $F / F_{q}$ is an automorphism $h$ of $F / F_{q}$ such that $h\left(x_{i}\right)$ is a conjugate of $x_{i}$ for each $i$.

Lemma 4.1. Let $L$ be an ordered oriented $m$-component link. We write $\pi=$ $\pi_{1}\left(E_{L}\right)$.

(1) Any $F / F_{q}$-structure $\varphi: \pi \rightarrow F / F_{q}$ induces an isomorphism $\pi / \pi_{q} \rightarrow F / F_{q}$.

(2) If $\varphi$ and $\varphi^{\prime}$ are $F / F_{q}$-structures for $L$, then there exists a special automorphism $\Theta$ such that $\varphi^{\prime}=\Theta \circ \varphi$. 
Proof. We denote by $\gamma: F \rightarrow \pi$ the map which sends $x_{i}$ to $\mu_{i}$. We also pick a map $\psi: F \rightarrow F$ such that the $\operatorname{map} F \stackrel{\psi}{\rightarrow} F \rightarrow F / F_{q}$ agrees with $\varphi \circ \gamma$. Finally note that $\gamma$ descends to a map $F / F_{q} \rightarrow \pi / \pi_{q}$ which we denote by $\bar{\gamma}$. We now have the following commutative diagram, where the vertical maps are the obvious projection maps:

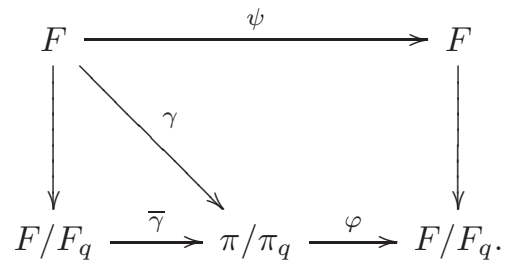

It follows from Stallings' theorem Sta65 applied to $\psi$ and from the commutativity of the diagram that the map $\varphi \circ \bar{\gamma}$ is an isomorphism. On the other hand it follows from Milnor's theorem [Mi57, Theorem 4] that $\bar{\gamma}$ is surjective. We now conclude that $\varphi$ is an isomorphism. This concludes the proof of (1).

The second statement is an immediate consequence of (1).

Note that it follows from Lemma 4.1 (1) and Mi57 that a link admits an $F / F_{q^{-}}$ structure if and only if Milnor's $\bar{\mu}$-invariants of the form $\bar{\mu}\left(i_{1} \cdots i_{q-1}\right)$ defined in Mi57 vanish for $L$.

4.2. Obstructions to links being concordant. Now we are ready to derive the following theorem:

Theorem 4.2. Suppose two m-component ordered links $L$ and $L^{\prime}$ are concordant, $\psi: \mathbb{Z}^{m} \rightarrow H$ is an admissible homomorphism, and $R$ is a subring of $\mathbb{C}$ closed under complex conjugation. If $\varphi$ and $\varphi^{\prime}$ are arbitrary $F / F_{q}$-structures for $L$ and $L^{\prime}$, respectively, then there exists a special automorphism $\Theta$ of $F / F_{q}$ such that for any $\alpha \in P_{k}\left(R, F / F_{q}\right)$ we have

$$
\operatorname{rank}(L, \psi, \alpha \circ \varphi)=\operatorname{rank}\left(L^{\prime}, \psi, \alpha \circ \Theta \circ \varphi^{\prime}\right)
$$

and

$$
\tau^{(\alpha \circ \varphi) \otimes \psi}(L)= \pm d h \cdot \tau^{\left(\alpha \circ \Theta \circ \varphi^{\prime}\right) \otimes \psi}\left(L^{\prime}\right) \in Q(H)^{\times} / N(Q(H))
$$

for some $d \in \operatorname{det}\left(\alpha\left(F / F_{q}\right)\right)$ and $h \in H$.

Proof. Let $W$ be the exterior of a concordance, $E=E_{L}$, and $E^{\prime}=E_{L^{\prime}}$. Since $H_{*}(E) \cong H_{*}(W) \cong H_{*}\left(E^{\prime}\right)$ we can apply Stallings' theorem [Sta65] to conclude that the inclusion maps induce isomorphisms

$$
\pi_{1}(E) / \pi_{1}(E)_{q} \stackrel{\cong}{\longrightarrow} \pi_{1}(W) / \pi_{1}(W)_{q} \cong \pi_{1}\left(E^{\prime}\right) / \pi_{1}\left(E^{\prime}\right)_{q} .
$$

Observe that the composition sends meridians of $L$ to (conjugates of) meridians of $L^{\prime}$. It follows that the composition

$$
\pi_{1}\left(E^{\prime}\right) \longrightarrow \pi_{1}\left(E^{\prime}\right) / \pi_{1}\left(E^{\prime}\right)_{q} \cong \pi_{1}(W) / \pi_{1}(W)_{q} \cong \pi_{1}(E) / \pi_{1}(E)_{q} \longrightarrow F / F_{q}
$$

gives an $F / F_{q}$-structure on $L^{\prime}$ which we denote by $\varphi^{\prime \prime}$.

For $\alpha \in P_{k}\left(R, F / F_{q}\right), \alpha \circ \varphi \in P_{k}\left(R, \pi_{1}(E)\right)$ corresponds to $\alpha \circ \varphi^{\prime \prime} \in P_{k}\left(R, \pi_{1}\left(E^{\prime}\right)\right)$ under the bijection $\Phi$ in Theorem 3.1, by the definition of $F / F_{q}$-concordance and the definition of $\Phi$ (see Subsection 3.1). Now by Theorem 3.1, we have

$$
\begin{gathered}
\operatorname{rank}(L, \psi, \alpha \circ \varphi)=\operatorname{rank}\left(L^{\prime}, \psi, \alpha \circ \varphi^{\prime \prime}\right) \\
\tau^{(\alpha \circ \varphi) \otimes \psi}(L)=\tau^{\left(\alpha \circ \varphi^{\prime \prime}\right) \otimes \psi}\left(L^{\prime}\right) .
\end{gathered}
$$

By Lemma 4.1 there exists a special automorphism $\Theta$ such that $\varphi^{\prime \prime}=\Theta \circ \varphi^{\prime}$. The theorem now immediately follow from these observations.

The following corollary is equivalent to Theorem 1.1 given in the introduction. 
Corollary 4.3. Suppose $L$ is an $m$-component slice link, $\psi: \mathbb{Z}^{m} \rightarrow H$ is an admissible homomorphism, and $R$ is a subring of $\mathbb{C}$ closed under complex conjugation. Then for any $\alpha \in P_{k}(R, \pi(L))$, we have $\operatorname{rank}(L, \psi, \alpha)=k(m-1)$ and

$$
\tau^{\alpha \otimes \psi}(L)= \pm d h \cdot \prod_{i=1}^{m} \operatorname{det}\left(\mathrm{id}-\alpha\left(\mu_{i}\right) t_{i}\right)^{-1} \in Q(H)^{\times} / N(Q(H))
$$

for some $d \in \operatorname{det}(\alpha(\pi(L)))$ and $h \in H$.

Proof. Write $\pi=\pi_{1}\left(E_{L}\right)$. Let $\alpha \in P_{k}(R, \pi(L))$. By definition $\alpha$ factors through a $p$-group. Since $p$-groups are nilpotent it follows that there exists a $q$ such that $\alpha$ factors through $\pi / \pi_{q}$. Since $L$ is slice, by the proof of Theorem 4.2 there exists an $F / F_{q}$-structure $\varphi: \pi \rightarrow \pi / \pi_{q} \cong F / F_{q}$ on $L$. In particular there exists $\alpha_{0} \in$ $P_{k}\left(R, F / F_{q}\right)$ such that $\alpha=\alpha_{0} \circ \varphi$. Since $L$ is concordant to the trivial link, we may by Theorem 4.2 assume without loss of generality that $L$ is already the trivial link. (Precisely speaking, this requires the change of $\alpha_{0}$ to $\alpha_{0} \circ \Theta$ for some special automorphism $\Theta$ of $F / F_{q}$, but this can be ignored since $\operatorname{det}\left(\right.$ id $\left.-\alpha\left(\mu_{i}\right) t_{i}\right)=$ $\operatorname{det}\left(\mathrm{id}-\alpha_{0}\left(x_{i}\right) t_{i}\right)$ is left unchanged when $x_{i}$ is replaced with its conjugate.) The corollary now follows from the explicit calculation for the unlink, which will be done in Theorem 6.2 .

\section{Representation theory of $p$-GROUPS}

In this section we collect a few basic facts of the theory of representations factoring through $p$-groups. This summary will be useful in the later discussion of examples.

Given $k$ we denote by $P(k) \subset \mathrm{GL}(\mathbb{C}, k)$ the subgroup of permutation matrices, i.e. matrices with exactly one non-trivial entry in each row and in each column, and all the non-trivial entries are equal to one. Let $R \subset \mathbb{C}$ be a subring which is closed under complex conjugation. We then write $D(R, k) \subset \mathrm{GL}(R, k)$ for the subgroup of diagonal matrices.

Proposition 5.1. Let $\pi$ be a group with generators $g_{1}, \ldots, g_{n}$. Let $\alpha: \pi \rightarrow \operatorname{GL}(\mathbb{C}, k)$ be a representation. We write $X_{i}=\alpha\left(g_{i}\right), i=1, \ldots, n$. Let $p$ be a prime. Then $\alpha$ factors through a $p$-group if and only if there exists a matrix $Q \in \mathrm{GL}(\mathbb{C}, k)$ such that for $i=1, \ldots, n$ we can write $Q X_{i} Q^{-1}=P_{i} D_{i}$ with $P_{i} \in P(k)$ and $D_{i} \in D(\mathbb{C}, k)$ such that

(1) $P_{1}, \ldots, P_{n}$ generate a subgroup of $p$-power order, and

(2) the diagonal entries of $D_{1}, \ldots, D_{n}$ are $p$-power roots of unity.

Proof. We will say that a matrix $D$ is $p$-diagonal if $D$ is diagonal and if all the diagonal entries are $p$-power roots of unity. We will several times make use of the following two basic observations:

(i) If $P, P^{\prime} \in P(k)$ and $D, D^{\prime} \in D(R, k)$ with $P D=P^{\prime} D^{\prime}$, then $P=P^{\prime}$ and $D=D^{\prime}$.

(ii) Let $P, P^{\prime} \in P(k)$ and $D, D^{\prime} \in D(R, k)$, then $P D P^{\prime} D^{\prime}=P P^{\prime} \cdot P^{\prime-1} D P^{\prime} D^{\prime}$ and $P^{\prime-1} D P^{\prime} D^{\prime}$ is a diagonal matrix, furthermore, if $D, D^{\prime}$ are $p$-diagonal, then $P^{\prime-1} D P^{\prime} D^{\prime}$ is also $p$-diagonal.

Now assume that we have a representation $\alpha: \pi \rightarrow \mathrm{GL}(\mathbb{C}, k)$ with the following property: there exists a matrix $Q \in \mathrm{GL}(\mathbb{C}, k)$ such that for $i=1, \ldots, n$ we can write $Q \alpha\left(g_{i}\right) Q^{-1}=P_{i} D_{i}$ with $P_{i} \in P(k), D_{i} \in D(\mathbb{C}, k)$ which satisfy conditions (1) and (2).

We claim that $\alpha(\pi)$ is a $p$-group. Without loss of generality we can assume that $Q$ is the identity. Let $Y$ be any element in the group $\alpha(\pi)$, we can write $Y=\left(P_{i_{1}} D_{i_{1}}\right)^{\epsilon_{1}} \ldots .\left(P_{i_{r}} D_{i_{r}}\right)^{\epsilon_{r}}$ for some $i_{j} \in\{1, \ldots, n\}, \epsilon_{j} \in\{ \pm 1\}$. We have to 
show that $Y$ has $p$-power order. Let $X=P_{i_{1}}^{\epsilon_{1}} \cdots P_{i_{r}}^{\epsilon_{r}}$. By assumption $X^{p^{s}}=\mathrm{id}$ for some $s$. Using the above observations we can write

$$
Y^{p^{s}}=\left(\left(P_{i_{1}} D_{i_{1}}\right)^{\epsilon_{1}} \cdots \cdot\left(P_{i_{r}} D_{i_{r}}\right)^{\epsilon_{r}}\right)^{p^{s}}=X^{p^{s}} \cdot D
$$

for some $p$-diagonal matrix $D$. It follows that $Y^{p^{s}}=D$ (and hence $Y$ ) has $p$-power order. This shows that $\alpha(\pi)$ is a $p$-group.

Now let $\alpha: \pi \rightarrow \mathrm{GL}(\mathbb{C}, k)$ be a representation which factors through a $p$-group $P$. We write $X_{i}=\alpha\left(g_{i}\right), i=1, \ldots, n$. By We03, Corollary 4.9] any representation $\alpha: P \rightarrow \mathrm{GL}(\mathbb{C}, k)$ of the $p$-group $P$ is induced from a representation of degree 1 . This means that there exists a finite index subgroup $Q \subset P$ and a one-dimensional representation $Q \rightarrow \mathrm{GL}(\mathbb{C}, 1)$ such that $\alpha$ is given by the natural $P$-left action on $\mathbb{C}[P] \otimes_{\mathbb{C}[Q]} \mathbb{C}$. Note that the one-dimensional representation is necessarily of $p$-power order. Now pick representatives $p_{1}, \ldots, p_{l}$ for $P / Q$. These representatives defines a basis for $\mathbb{C}[P] \otimes_{\mathbb{C}[Q]} \mathbb{C}$, and writing $\alpha$ with respect to this basis we see that $\alpha$ is of the required type.

\section{BOUNDARY LINKS}

Boundary links form an important subclass of links which has been intensely studied over the years. In this section we will show how to calculate twisted invariants for boundary links and we will study the twisted invariants of boundary links that are 'boundary slice'.

\subsection{Invariants of boundary links.}

Definition. A boundary link is an $m$-component oriented link in $S^{3}$ which has $m$ disjoint Seifert surfaces, i.e. there exist $m$ disjoint oriented surfaces $V_{1}, \ldots, V_{m} \subset S^{3}$ such that $\partial\left(V_{i}\right)=L_{i}, i=1, \ldots, m$. We refer to such a disjoint collection of Seifert surfaces as a boundary link Seifert surface.

Remark. Not every link is a boundary link. For example it is clear that a boundary link has trivial linking numbers. Furthermore, Milnor's Mi57] $\bar{\mu}$-invariants are trivial for a boundary link, and in fact trivial for any link concordant to a boundary link. Cochran and Orr CO90, CO93 showed that there also exist links with vanishing $\bar{\mu}$-invariants which are not concordant to a boundary link (cf. also GL92 and [Le94]).

Throughout this section let $F$ be the free group on the generators $x_{1}, \ldots, x_{m}$. An $F$-link is a pair $(L, \varphi)$ where $L$ is an oriented $m$-component link in $S^{3}$ and $\varphi: \pi(L) \rightarrow F$ is an epimorphism sending an $i^{\text {th }}$ meridian to $x_{i}$. Note that $\varphi$ gives rise to a map from $E_{L}$ to the wedge of $m$ circles, and by a standard transversality argument such a map then gives rise to a boundary link Seifert surface. Conversely, the existence of a boundary link Seifert surface $V$ for $L$ produces such an epimorphism $\pi(L) \rightarrow F$ by the Thom-Pontryagin construction.

Given an oriented boundary link $L$ with disjoint oriented surfaces $V_{1}, \ldots, V_{m} \subset$ $S^{3}$ we can define the 'boundary link Seifert matrix' as follows: Let $g_{i}$ be the genus of $V_{i}$. For $i=1, \ldots, m$ pick a basis $v_{i, 1}, \ldots, v_{i, 2 g_{i}}$ for $H_{1}\left(V_{i}\right)$. For $k, l \in\{1, \ldots, m\}$ we define $A_{k l}$ to be the $2 g_{k} \times 2 g_{l}$-matrix given by $\left(A_{i j}\right)_{k l}=\operatorname{lk}\left(v_{i, k},\left(v_{j, l}\right)_{+}\right)$where $\left(v_{j, l}\right)_{+}$denotes the positive push-off of $v_{j, l}$ from $V_{j}$ into $S^{3} \backslash V$. We now view $\left(A_{i j}\right)_{k l}$ as an $m \times m$-matrix of matrices and we refer to it as a boundary link Seifert matrix. We refer to [Lia77] and [Ko85, Ko87] for details and for more information on boundary link Seifert matrices.

We now turn to the computation of twisted invariants of boundary links. Let $(L, \varphi)$ be an $F$-link and $A=\left(A_{i j}\right)$ a corresponding boundary link Seifert matrix 
where $A_{i j}$ is an $r_{i} \times r_{j}$-matrix. Let $r=\sum_{i=1}^{m} r_{i}$ and

$$
T:=\operatorname{diag}(\underbrace{t_{1}, \ldots, t_{1}}_{r_{1}}, \ldots, \underbrace{t_{m}, \ldots, t_{m}}_{r_{m}}) .
$$

We view $A^{t}-A T$ as an $r \times r$-matrix over $R\left[t_{1}^{ \pm 1}, \ldots, t_{m}^{ \pm 1}\right]$. Let $\psi: \mathbb{Z}^{m} \rightarrow H$ be an admissible epimorphism to a nontrivial free abelian group $H$ and let $\alpha: F \rightarrow$ $\operatorname{GL}(R, k)$ be a representation. Note that all entries of the matrix $(\alpha \otimes \psi)\left(A^{t}-A T\right)$ are sums of monomials in one variable, we can therefore unambiguously define the matrix $(\alpha \otimes \psi)\left(A^{t}-A T\right)$ to be the $r k \times r k$-matrix over $R[H]$ given by replacing each summand $a t_{i}^{j}$ of an entry of $A^{t}-A T$ by the matrix $a \alpha\left(x_{i}\right)^{j} \psi\left(t_{i}\right) \in \operatorname{GL}(R[H], k)$. (Here and throughout this section we will identify the additive group $\mathbb{Z}^{m}$ with the multiplicative group generated by $t_{1}, \ldots, t_{m}$.)

Theorem 6.1. Let $(L, \varphi)$ be an F-link. Let $A$ be a boundary link Seifert matrix corresponding to Seifert surfaces given by $\varphi$. Let $\psi: \pi(L) \rightarrow H$ be an admissible epimorphism to a nontrivial free abelian group $H$. Let $R \subset \mathbb{C}$ be a subring closed under complex conjugation, and let $\alpha: \pi(L) \rightarrow \mathrm{GL}(R, k)$ be a unitary representation which factors through the epimorphism $\varphi$. Then the following hold:

(1) We have $\operatorname{rank}(L, \psi, \alpha) \geq k(m-1)$ and equality holds if and only if $\operatorname{det}((\alpha \otimes$ $\left.\psi)\left(A^{t}-A T\right)\right) \neq 0$,

(2) If $\operatorname{rank}(L, \psi, \alpha)=k(m-1)$, then

$$
\tau^{\alpha \otimes \psi}(L) \doteq \operatorname{det}\left((\alpha \otimes \psi)\left(A^{t}-A T\right)\right) \cdot \prod_{i=1}^{m} \operatorname{det}\left(\mathrm{id}-\alpha\left(x_{i}\right) t_{i}\right)^{-1} \in \frac{Q(H)^{\times}}{N(Q(H))} .
$$

(3) If $\alpha$ factors through a $p$-group, then $\operatorname{rank}(L, \psi, \alpha)=k(m-1)$.

Proof. Let $V_{1} \cup \cdots \cup V_{m} \subset E_{L}$ be a properly embedded boundary link Seifert surface for $L$ corresponding to $\varphi$ together with bases $v_{i, 1}, \ldots, v_{i, 2 g_{i}}$ for $H_{1}\left(V_{i}\right)$ such that $\left(A_{i j}\right)$ is the corresponding Seifert matrix, i.e. $\left(A_{i j}\right)_{k l}=\operatorname{lk}\left(v_{i, k},\left(v_{j, l}\right)_{+}\right)$. Let $C=S^{3} \backslash\left(\nu V_{1} \cup \cdots \cup \nu V_{m}\right)$. Note that the induced maps $\pi_{1}\left(V_{i}\right) \rightarrow F, i=1, \ldots, m$ and $\pi_{1}(C) \rightarrow F$ are trivial, in particular $\alpha$ and $\psi$ restricted to $\pi_{1}\left(V_{i}\right), i=1, \ldots, m$ and $\pi_{1}(C)$ are trivial. We therefore get the following exact Mayer-Vietoris sequence:

$$
\begin{aligned}
& \bigoplus_{i=1}^{m} H_{1}\left(V_{i}\right) \otimes_{\mathbb{Z}} R[H]^{k} \longrightarrow H_{1}(C) \otimes_{\mathbb{Z}} R[H]^{k} \longrightarrow H_{1}\left(E_{L} ; R[H]^{k}\right) \\
& \quad \longrightarrow \bigoplus_{i=1}^{m} H_{0}\left(V_{i}\right) \otimes_{\mathbb{Z}} R[H]^{k} \longrightarrow H_{0}(C) \otimes_{\mathbb{Z}} R[H]^{k} \longrightarrow H_{0}\left(E_{L} ; R[H]^{k}\right) \longrightarrow 0
\end{aligned}
$$

(We refer to [FK06, Section 3] for details.) Now let $c_{i, 1}, \ldots, c_{i, 2 g_{i}}, i=1, \ldots, m$ be the basis for $H_{1}(C)$ dual to the basis $v_{i, 1}, \ldots, v_{i, 2 g_{i}}, i=1, \ldots, m$, i.e. $\operatorname{lk}\left(c_{i, j}, v_{k, l}\right)=$ $\delta_{i k} \cdot \delta_{j l}$. Using these bases it is well-known that the map

$$
H_{1}\left(V_{i}\right) \otimes_{\mathbb{Z}} R[H]^{k} \longrightarrow H_{1}(C) \otimes_{\mathbb{Z}} R[H]^{k}
$$

is represented by $(\alpha \otimes \psi)\left(A^{t}-A T\right)$. We write $n=\sum_{i=1}^{m} 2 g_{i} k$. Note that $(\alpha \otimes$ $\psi)\left(A^{t}-A T\right)$ is an $n \times n$-matrix. We now have

$$
\begin{aligned}
\operatorname{rank}(L, \psi, \alpha)= & \operatorname{dim}_{Q(H)} H_{1}\left(E_{L} ; Q(H)^{k}\right) \\
= & \operatorname{dim}\left(\bigoplus_{i=1}^{m} H_{0}\left(V_{i}\right) \otimes_{\mathbb{Z}} Q(H)^{k}\right)-\operatorname{dim}\left(H_{0}(C) \otimes_{\mathbb{Z}} Q(H)^{k}\right) \\
& \quad+\operatorname{dim}\left(H_{0}\left(E_{L} ; Q(H)^{k}\right)+\operatorname{dim}_{Q(H)}\left(H_{1}(C) \otimes Q(H)^{k}\right)\right. \\
& \quad-\operatorname{rank}\left((\alpha \otimes \psi)\left(A^{t}-A T\right)\right) \\
= & k m-k+0+n-\operatorname{rank}\left((\alpha \otimes \psi)\left(A^{t}-A T\right)\right) .
\end{aligned}
$$


In particular $\operatorname{rank}(L, \psi, \alpha) \geq k m-k$, and equality holds if and only if the rank of the $n \times n$-matrix $(\alpha \otimes \psi)\left(A^{t}-A T\right)$ is equal to $n$. This proves (1).

We now turn to the calculation of $\tau^{\alpha \otimes \psi}(L)$. Suppose that $\operatorname{rank}(L, \psi, \alpha)=k(m-$ $1)$. For $i=1, \ldots, m$ we pick a point $u_{i} \in V_{i}$. Note that $\left\{u_{i},\left\{v_{i, 1}, \ldots, v_{i, 2 g_{i}}\right\}\right\}$ defines a basis for the free $\mathbb{Z}$-module $H_{*}\left(V_{i}\right)$ and

$$
\tau\left(V_{i} ;\left\{u_{i},\left\{v_{1}^{i}, \ldots, v_{2 g_{i}}^{i}\right\}\right\}\right)= \pm 1 \in \mathbb{Z}
$$

since \pm 1 are the only units in the ring $\mathbb{Z}$. We now denote by $e_{1}, \ldots, e_{k}$ the standard basis of $Q^{k}$. For $i=1, \ldots, m$ we then let

$$
\begin{aligned}
& \mathcal{V}_{i 0}=\left\{u_{i} \otimes e_{j}\right\}_{j=1, \ldots, k}, \\
& \mathcal{V}_{i 1}=\left\{v_{i, j} \otimes e_{l}\right\}_{j=1, \ldots, 2 g_{i}, l=1, \ldots, k} .
\end{aligned}
$$

Note that for $i=1, \ldots, m$ the set $\left\{\mathcal{V}_{i 0}, \mathcal{V}_{i 1}\right\}$ defines a basis for the free $Q(H)-$ module $H_{*}\left(V_{i}\right) \otimes_{\mathbb{Z}} Q(H)^{k}$ and

$$
\tau\left(V_{i} ;\left\{\mathcal{V}_{i 0}, \mathcal{V}_{i 1}\right\}\right)=\tau\left(V_{i} ;\left\{u_{i},\left\{v_{i, 1}, \ldots, v_{i, 2 g_{i}}\right\}\right\}\right)^{k}= \pm 1 \in \mathbb{Z}
$$

We now also pick a point $b \in C$ and for $i=1, \ldots, m-1$ we denote by $S_{i}$ the result of gluing $V_{i} \times-1$ and $V_{i} \times 1$ together along $\partial V_{i} \times[-1,1]$. Note that $S_{1}, \ldots, S_{m-1}$ are a basis for $H_{2}(C)$. We now let

$$
\begin{aligned}
& \mathcal{C}_{0}=\left\{p \otimes e_{j}\right\}_{j=1, \ldots, k}, \\
& \mathcal{C}_{1}=\left\{c_{i, j} \otimes e_{l}\right\}_{i=1, \ldots, m, j=1, \ldots, 2 g_{i}, l=1, \ldots, k}, \\
& \mathcal{C}_{2}=\left\{S_{i} \otimes e_{j}\right\}_{i=1, \ldots, m-1, j=1, \ldots, k} .
\end{aligned}
$$

Note that the set $\left\{\mathcal{C}_{0}, \mathcal{C}_{1}, \mathcal{C}_{2}\right\}$ defines a basis for the free $Q(H)$-module $H_{*}(C) \otimes_{\mathbb{Z}}$ $Q(H)^{k}$ and a similar argument as above shows that

$$
\tau\left(C ;\left\{\mathcal{C}_{0}, \mathcal{C}_{1}, \mathcal{C}_{2}\right\}\right)= \pm 1 \in \mathbb{Z}
$$

Now note that $\operatorname{rank}(L, \psi, \alpha)=k(m-1)$ implies by (1) that the map

$$
\bigoplus_{i=1}^{m} H_{1}\left(V_{i}\right) \otimes_{\mathbb{Z}} Q(H)^{k} \longrightarrow H_{1}(C) \otimes_{\mathbb{Z}} Q(H)^{k}
$$

is injective. This implies that the map

$$
H_{2}(C) \otimes Q(H)^{k} \longrightarrow H_{2}\left(E_{L} ; Q(H)^{k}\right)
$$

is an isomorphism, and we denote by $\mathcal{B}_{2}$ be the image of $\mathcal{C}_{2}$ under this isomorphism. By a slight abuse of notation we also write $\mathcal{C}_{2}:=\left\{S_{i} \otimes e_{j}\right\}_{i=1, \ldots, m-1, j=1, \ldots, k}$.

For $i=1, \ldots, m$ we now write $P_{i}=\mathrm{id}-\alpha\left(x_{i}\right) t_{i}$. Furthermore for $i=1, \ldots, m$ we let $\mu_{i}$ be a meridian of $L_{i}$ which is based at $b$ and which intersects $V_{i}$ geometrically once in the positive direction and which is disjoint from $V_{j}, j \neq i$.

We now denote by $p: \widetilde{E_{L}} \rightarrow E_{L}$ the $F$-cover corresponding to $\varphi$. We pick a point $\tilde{b}$ over $b$ and for $i=1, \ldots, m$ we denote by $\tilde{\mu}_{i}$ the component of $p^{-1}\left(\mu_{i}\right)$ such that $\partial \tilde{\mu}_{i}=\tilde{b}-x_{i} \cdot \tilde{b}$.

Claim. For $i \in\{1, \ldots, m-1\}$ and $j \in\{1, \ldots, k\}$ we define

$$
b_{i j}^{\prime}:=\tilde{\mu}_{i} \otimes P_{i}^{-1} e_{j}-\tilde{\mu}_{i+1} \otimes P_{i+1}^{-1} e_{j} .
$$

Then the following hold:

(1) $\left\{b_{i j}^{\prime}\right\}$ is a basis for $H_{1}\left(E_{L} ; Q(H)^{k}\right)$,

(2) for any $i, j, k$ and $l$ we have

$$
b_{i j}^{\prime} \cdot\left(S_{r} \otimes e_{s}\right)= \begin{cases}\delta_{j s}, & \text { if } r=i \\ -\delta_{j s}, & \text { if } r=i+1 .\end{cases}
$$


(3) for any $i$ and $j$ we have

$$
r\left(b_{i j}^{\prime}\right)=u_{i} \otimes P_{i}^{-1} e_{j}-u_{i+1} \otimes P_{i+1}^{-1} e_{j} .
$$

In order to prove the claim, first note that

$$
\begin{aligned}
\partial\left(\tilde{\mu}_{i} \otimes e_{j}\right) & =\left(1-x_{i}\right) \tilde{b} \otimes e_{j} \\
& =\tilde{b} \otimes(\alpha \otimes \psi)\left(1-x_{i}\right) e_{j} \\
& =\tilde{b} \otimes\left(\operatorname{id}-\alpha\left(x_{i}\right) t_{i}\right) e_{j}=\tilde{b} \otimes P_{i} e_{j} .
\end{aligned}
$$

It therefore follows that

$$
\partial\left(b_{i j}^{\prime}\right)=\tilde{b} \otimes P_{i} P_{i}^{-1} e_{j}-\tilde{b} \otimes P_{i+1} P_{i+1}^{-1} e_{j}=0 .
$$

The calculation of $b_{i j}^{\prime} \cdot\left(S_{r} \otimes e_{s}\right)$ follows easily from the definitions. This shows in particular that the $b_{i j}^{\prime}$ are linearly independent and hence form a basis for $H_{1}\left(E_{L} ; Q(H)^{k}\right)$ Finally it is straightforward to verify that

$$
r\left(b_{i j}^{\prime}\right)=u_{i} \otimes P_{i}^{-1} e_{j}-u_{i+1} \otimes P_{i+1}^{-1} e_{j}
$$

This concludes the proof of the claim.

For $i=1, \ldots, m-1$ and $j=1, \ldots, k$ we now let

$$
b_{i j}=\sum_{r=i}^{m-1} b_{r j}^{\prime} .
$$

We write $\mathcal{C}_{1}:=\left\{b_{i j}\right\}$. It follows immediately from the above claim that $\mathcal{C}_{1}$ is a basis, and that $\mathcal{C}_{1}$ is dual to $\mathcal{C}_{2}$. We now consider the following short exact sequence of $Q(H)$-complexes:

$$
0 \longrightarrow \bigoplus_{i=1}^{m} C_{*}\left(V_{i} ; Q(H)^{k}\right) \longrightarrow C_{*}\left(C ; Q(H)^{k}\right) \longrightarrow C_{*}\left(E_{L} ; Q(H)^{k}\right) \longrightarrow 0
$$

together with the above bases. It follows from Theorem 2.2 (4) together with the above calculations and from the definition of torsion that

$$
\tau^{\alpha \otimes \psi}\left(L,\left\{\mathcal{B}_{1}, \mathcal{B}_{2}\right\}\right)=\operatorname{det}\left((\alpha \otimes \psi)\left(A^{t}-A T\right)\right) \cdot \tau(C),
$$

where $C$ is the following complex with the canonical bases:

$$
\begin{array}{r}
0 \longrightarrow Q(H)^{k(m-1)} \stackrel{f}{\longrightarrow} Q(H)^{k m} \stackrel{g}{\longrightarrow} Q(H)^{k} \longrightarrow 0 \\
f=\left(\begin{array}{cccc}
P_{1}^{-1} & 0 & \ldots & 0 \\
-P_{2}^{-1} & P_{2}^{-1} & \ldots & 0 \\
0 & \ddots & \ddots & \vdots \\
0 & \ldots & -P_{m-1}^{-1} & P_{m-1}^{-1} \\
0 & \ldots & 0 & -P_{m}^{-1}
\end{array}\right) \\
g=\left(\begin{array}{llll}
P_{1} & \ldots & P_{m}
\end{array}\right)
\end{array}
$$

It now follows (cf. e.g. TTu01, Theorem 2.2]) that

$$
\tau(C)=\prod_{i=1}^{m} \operatorname{det}\left(P_{i}\right)^{-1}
$$

This concludes the proof of the second statement of the theorem.

Finally note that $\operatorname{det}\left(A-A^{t}\right)= \pm 1$ (see Ko87]), in particular if $\alpha$ factors through a $p$-group, then it follows immediately from Theorem 3.2 that $\operatorname{det}\left((\alpha \otimes \psi)\left(A^{t}-\right.\right.$ $A T)) \neq 0$. This concludes the proof of part (3) of the theorem.

The following corollary follows immediately from taking $A$ to be the trivial matrix. 
Corollary 6.2. Let $L \subset S^{3}$ be the $m$-component unlink in $S^{3}$ with meridians $\mu_{1}, \ldots, \mu_{m}$. Let $\psi: \mathbb{Z}^{m} \rightarrow H$ be an admissible homomorphism. Let $R \subset \mathbb{C}$ be a subring closed under complex conjugation, and let $\alpha: \pi(L) \rightarrow \operatorname{GL}(R, k)$ be a unitary representation. Then

$$
\operatorname{rank}(L, \psi, \alpha)=k(m-1)
$$

and

$$
\tau^{\alpha \otimes \psi}(L)= \pm d h \cdot \prod_{i=1}^{m} \operatorname{det}\left(\operatorname{id}-\psi\left(\mu_{i}\right) \alpha\left(\mu_{i}\right)\right)^{-1} \in Q(H)^{\times} / N(Q(H))
$$

with $d \in \operatorname{det}(\alpha(\pi(L)))$ and $h \in H$.

6.2. Boundary slice links. Let $L \subset S^{3}$ be an $m$-component boundary link. We say $L$ is boundary slice if there exist disjointly embedded 3 -manifolds $W_{1}, \ldots, W_{m} \in$ $D^{4}$ such that for $i=1, \ldots, m$, the boundary $\partial W_{i}$ is the union of a Seifert surface and a slice disk for $L_{i}$. It is known that a boundary slice link is boundary slice with respect to any boundary link Seifert surface. We note that boundary slice links are slice; it is a long-standing open question whether the converse holds for boundary links.

Obstructions to being boundary slice has been studied by several authors, including Cappell and Shaneson [CS80, Duval [Du86], Ko Ko87, Mio Mi87, Sheiham [Sh03, Sh06]. The following torsion obstruction to being boundary slice is a consequence of these works.

Theorem 6.3. Let $(L, \varphi)$ be an m-component F-link which is boundary slice. Let $\alpha: \pi(L) \stackrel{\varphi}{\rightarrow} F \rightarrow \mathrm{GL}(R, k)$ be a unitary representation where $R \subset \mathbb{C}$ is a subring closed under complex conjugation. Let $\psi: \mathbb{Z}^{m} \rightarrow H$ be an admissible homomorphism. If $\operatorname{rank}(L, \psi, \alpha)=k(m-1)$, then

$$
\tau^{\alpha \otimes \psi}(L)= \pm d h \cdot \prod_{i=1}^{m} \operatorname{det}\left(\operatorname{id}-\psi\left(\mu_{i}\right) \alpha\left(\mu_{i}\right)\right)^{-1} \in Q(H)^{\times} / N(Q(H))
$$

for some $d \in \operatorname{det}(\alpha(\pi(L)))$ and $h \in H$.

Proof. Let $V=V_{1} \cup \cdots \cup V_{m}$ be a Seifert surface for $L$ corresponding to $\varphi$ and let $\left(A_{i j}\right)$ be the corresponding boundary link Seifert matrix. We denote by $g_{i}$ the genus of $V_{i}$. By the first remark of this subsection, there are disjointly embedded 3-manifolds $W_{i}$ in $D^{4}$ such that $\partial W_{i}=V_{i} \cup\left(\right.$ a slice disk for $\left.L_{i}\right)$. By Ko Ko87, Lemma 3.3], it follows that $\left(A_{i j}\right)$ is metabolic. This means that for $i=1, \ldots, m$ there exists an invertible $2 g_{i} \times 2 g_{i}$ matrix $P_{i}$ such that each $P_{i} A_{i j} P_{j}^{t}$ is of the form

$$
\left(\begin{array}{cc}
0 & C \\
D & E
\end{array}\right)
$$

where 0 is a $g_{i} \times g_{j}$-matrix. (In fact Ko Ko87 has shown that in higher odd dimensions $A$ being metabolic is in fact a sufficient condition for being boundary slice.) It follows easily that $(\alpha \otimes \psi)\left(A^{t}-A T\right)$ is equivalent to a matrix of the form

$$
\pm h\left(\begin{array}{cc}
0 & X \\
X^{t} & Y
\end{array}\right)
$$

where $h \in H$ and $X$ and $Y$ are matrices over $R[H]$ of size $k \sum_{i} g_{i}$. It now follows that

$$
\operatorname{det}\left((\alpha \otimes \psi)\left(A^{t}-A T\right)\right)= \pm u \operatorname{det}(R) \cdot \operatorname{det}\left(\bar{R}^{t}\right)= \pm u \operatorname{det}(R) \cdot \overline{\operatorname{det}(R)}
$$

for some $u \in H$. The theorem now follows immediately from Theorem 6.1 and the assumption that $\operatorname{rank}(L, \psi, \alpha)=k(m-1)$. 
Remark. Let $L$ be a boundary link. According to Theorem 4.2 the twisted torsion corresponding to any representation factoring through a $p$-group gives a sliceness obstruction. On the other hand, according to Theorems 6.1 and 6.3 the twisted torsions corresponding to many more representations give an obstruction to being boundary slice. This is very similar to the situation in Fr05 where the vanishing of the eta invariant for (boundary) slice links is investigated, as well as CKo99, Sm89] (cf. [Fr05, Theorem 4.8] and [Fr05, Theorem 4.3]). Levine [Le07] used work of Vogel Vo90] to resolve the apparent discrepancy in [Fr05] between the vanishing of the eta invariants for boundary links which are slice and those which are boundary slice.

\section{Computation FOR SATEllite Links}

7.1. The satellite construction. Let $L \subset S^{3}$ be an $m$-component oriented link and let $K \subset S^{3}$ be a knot. Let $A \subset E_{L}$ be a simple closed curve, unknotted in $S^{3}$. Then $S^{3} \backslash \nu A$ is a solid torus. Let $\phi: \partial(\overline{\nu A}) \rightarrow \partial(\overline{\nu K})$ be a diffeomorphism which sends a meridian of $A$ to a longitude of $K$, and a longitude of $A$ to a meridian of $K$. The space

$$
\left(S^{3} \backslash \nu A\right) \cup_{\phi}\left(S^{3} \backslash \nu K\right)
$$

is diffeomorphic to $S^{3}$. The image of $L$ is denoted by $S=S(L, K, A)$. We say $S$ is the satellite link with companion $K$, orbit $L$ and axis $A$. Put differently, $S$ is the result of replacing a tubular neighborhood of $K$ by a link in a solid torus, namely by $L \subset S^{3} \backslash \nu A$. Note that $S$ inherits an orientation from $L$.

An important example is given by letting $L$ be the unlink with two components and $A \subset E_{L}$ as in Figure 1. The corresponding satellite knot $S(L, K, A)$ is called

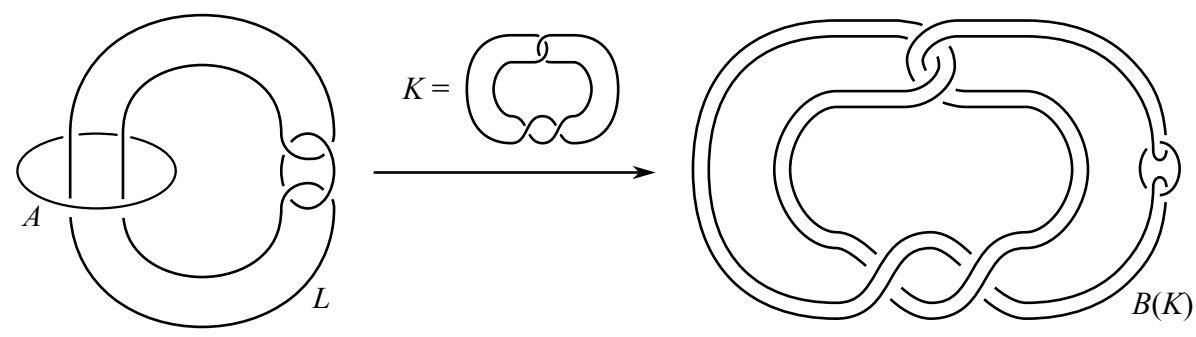

Figure 1. The Bing double of the Figure 8 knot.

the Bing double of $K$ and referred to as $B(K)$.

We now return to the discussion of satellite links in general. Note that the abelianization map $\pi_{1}\left(S^{3} \backslash \nu K\right) \rightarrow \mathbb{Z}$ gives rise to a map of degree one $S^{3} \backslash \nu K$ to $\overline{\nu A}$ which is a diffeomorphism on the boundary. In particular we get an induced map

$$
E_{S}=\left(S^{3} \backslash \nu A \backslash \nu L\right) \cup_{\phi}\left(S^{3} \backslash \nu K\right) \longrightarrow\left(S^{3} \backslash \nu A \backslash \nu L\right) \cup \overline{\nu A}=E_{L}
$$

which we denote by $f$. Note that $f$ is a diffeomorphism on the boundary and that $f$ induces an isomorphism of homology groups.

Before we state the next lemma note that the curve $A$ defines an element in $A \in \pi(L)$ which is well-defined up to conjugation.

Lemma 7.1. Let $L, K, A, S=S(L, K, A)$ and $f$ as above. Let $Q$ be a subfield of $\mathbb{C}$ and let $\alpha: \pi(L) \rightarrow \mathrm{GL}(Q, k)$ be a unitary representation. We denote the representation $\pi(S) \stackrel{f}{\rightarrow} \pi(L) \stackrel{\alpha}{\rightarrow} \operatorname{GL}(Q, k)$ by $\alpha$ as well. Let $\psi: \mathbb{Z}^{m} \rightarrow H$ be an admissible homomorphism such that $\psi(A)=0$. Denote by $z_{1}, \ldots, z_{k}$ the eigenvalues 
of $\alpha(A)$ and let $\Delta_{K} \in \mathbb{Z}\left[t^{ \pm 1}\right]$ be a fixed representative of the Alexander polynomial of $K$. Then the following hold:

(1) $\operatorname{rank}(S, \psi, \alpha)=\operatorname{rank}(L, \psi, \alpha)$ if and only if $\Delta_{K}\left(z_{i}\right) \neq 0$ for $i=1, \ldots, k$,

(2) if $\Delta_{K}\left(z_{i}\right) \neq 0$ for $i=1, \ldots, k$, then

$$
\tau^{\alpha \otimes \psi}(S)=\tau^{\alpha \otimes \psi}(L) \cdot \prod_{i=1}^{k} \Delta_{K}\left(z_{i}\right) \in Q(H)^{\times} / N(Q(H))
$$

up to multiplication by an element of the form \pm dh with $d \in \operatorname{det}(\alpha(\pi(L)))$ and $h \in H$.

Before we give the proof of Lemma 7.1 we first state and prove the following result which is an immediate consequence of Lemma 7.1 and Theorem 1.1

Corollary 7.2. Let $L$ be an oriented $m$-component slice link and let $K, A$ and $S=S(L, K, A)$ as above. Let $Q$ be a subfield of $\mathbb{C}$ closed under complex conjugation which has the unique factorization property and let $\alpha: \pi(L) \rightarrow \operatorname{GL}(R, k)$ be a representation which factors through a p-group. Let $\psi: \mathbb{Z}^{m} \rightarrow H$ be an admissible homomorphism. Denote by $z_{1}, \ldots, z_{k}$ the eigenvalues of $\alpha(A)$ and let $\Delta_{K} \in \mathbb{Z}\left[t^{ \pm 1}\right]$ be a fixed representative of the Alexander polynomial of $K$. Assume that $\psi(A)=0$. If $S=S(L, K, A)$ is slice, then $\Delta_{K}\left(z_{i}\right) \neq 0$ for $i=1, \ldots, k$ and

$$
\prod_{i=1}^{k} \Delta_{K}\left(z_{i}\right)= \pm d \cdot q \bar{q} \in Q
$$

for some $d \in \operatorname{det}(\alpha(\pi(L)))$ and $q \in Q$.

Proof of Lemma 7.1. We write $T=\overline{\nu A}$. Consider the following commutative diagram of short exact sequences of cellular chain complexes (where we write $\left.V=Q(H)^{k}\right)$ :

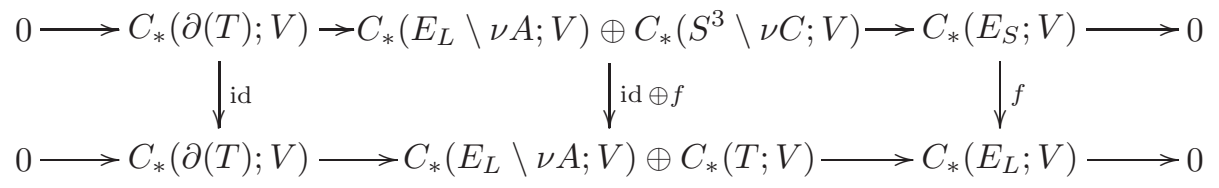

We assumed that $\psi(A)=0$. It follows that the map $\psi$ restricted to $T$ and restricted to $S^{3} \backslash \nu K$ is trivial. In particular $C_{*}^{\alpha \otimes \psi}\left(E_{K} ; Q(H)^{k}\right)=C_{*}^{\alpha}\left(E_{K} ; Q^{k}\right) \otimes Q(H)$ and $C_{*}^{\alpha \otimes \psi}\left(T ; Q(H)^{k}\right)=C_{*}^{\alpha}\left(T ; Q^{k}\right) \otimes Q(H)$. It is a consequence of Go78, Section 5] that $f_{*}: H_{1}\left(E_{K} ; Q^{k}\right) \rightarrow H_{1}\left(T ; Q^{k}\right)$ is always surjective and it is an isomorphism if and only if $\Delta_{K}\left(z_{i}\right) \neq 0$ for $i=1, \ldots, k$. The first statement of the lemma now follows immediately from the commutative diagram of long exact homology sequences corresponding to the above diagram.

Now suppose that $\Delta_{K}\left(z_{i}\right) \neq 0$ for $i=1 \ldots, k$.

Claim. The map $f_{*}: H_{j}\left(E_{K} ; Q^{k}\right) \rightarrow H_{j}\left(T ; Q^{k}\right)$ is an isomorphism for any $j$.

We already saw above that $f_{*}: H_{1}\left(E_{K} ; Q^{k}\right) \rightarrow H_{1}\left(T ; Q^{k}\right)$ is an isomorphism. Note that $E_{K}$ is homotopy equivalent to a 2 -complex and that $T$ is homotopy equivalent to 1 -complex. In particular $H_{2}\left(T ; Q^{k}\right)=0$ and it follows from the long exact sequence that $H_{2}\left(E_{K} ; Q^{k}\right)=0$ as well. Note that $\pi_{1}\left(E_{K}\right) \rightarrow \pi_{1}(T)$ is surjective, it follows that $f_{*}: H_{0}\left(E_{K} ; Q^{k}\right) \rightarrow H_{0}\left(T ; Q^{k}\right)$ is surjective as well. On the other hand we have that $\chi\left(E_{K}\right)=0=\chi(T)$, hence

$$
b_{0}\left(E_{K} ; Q^{k}\right)=b_{1}\left(E_{K} ; Q^{k}\right)+k \chi\left(E_{K}\right)=b_{1}\left(T ; Q^{k}\right)+k \chi(T)=b_{0}\left(T ; Q^{k}\right),
$$

i.e. $f_{*}: H_{0}\left(E_{K} ; Q^{k}\right) \rightarrow H_{0}\left(T ; Q^{k}\right)$ is in fact an isomorphism. This concludes the proof of the claim. 
Let $\mathcal{D}_{i}$ be $Q$-bases for $H_{i}\left(T ; Q^{k}\right), i=0,1$. We endow $H_{i}\left(E_{K} ; Q^{k}\right)$ with the corresponding basis $f_{*}^{-1}\left(\mathcal{D}_{i}\right), i=0,1$. It follows from the long exact sequence corresponding to $f: E_{K} \rightarrow T$ that $H_{*}\left(f: E_{K} \rightarrow T ; Q^{k}\right)=0$. It follows from Theorem 2.2 (4) that

$$
\tau^{\alpha}\left(T ;\left\{\mathcal{D}_{i}\right\}_{i=0,1}\right)=\tau^{\alpha}\left(E_{K},\left\{f_{*}^{-1}\left(\mathcal{D}_{i}\right)\right\}_{i=0,1}\right) \cdot \tau^{\alpha}\left(f: E_{K} \longrightarrow T\right) .
$$

(Here and in the following lines all equalities of torsion are up to multiplication by an element of the form $\pm d h$ with $d \in \operatorname{det}(\alpha(\pi(L)))$ and $h \in H$.) Note that the $\mathcal{D}_{i}$ are also $Q$-bases for $H_{i}\left(T ; Q(H)^{k}\right)=H_{i}\left(T ; Q^{k}\right) \otimes Q(H), i=0,1$. In particular the calculations in the previous lines also work for the torsions corresponding to twisting by $\alpha \otimes \psi$.

Let $B$ be a Seifert matrix for the knot $K$. It follows easily from Go78, Section 5] and [Tu01, Theorem 2.2] that

$$
\tau^{\alpha}\left(f: E_{K} \longrightarrow T\right)^{-1}=\prod_{i=1}^{k} \operatorname{det}\left(B^{t}-B z_{i}\right)=\prod_{i=1}^{k} \Delta_{K}\left(z_{i}\right) .
$$

Now pick a $Q(H)$-basis $\mathcal{B}$ for $H_{1}\left(E_{L} ; V\right)$ and denote the dual basis for $H_{2}\left(E_{L} ; V\right)$ by $\mathcal{B}^{\prime}$. Finally pick bases $\mathcal{E}_{i}, i=0,1,2$ for $H_{i}\left(\partial T ; Q(H)^{k}\right)$. Note that the bases $\mathcal{D}_{i}, \mathcal{B}, \mathcal{E}_{i}$ give rise to bases $\mathcal{F}_{i}, i=0,1,2$ for $H_{*}\left(E_{L} \backslash \operatorname{int} T ; Q(H)^{k}\right)$. It now follows from Theorem 2.2 (4) that

$$
\begin{aligned}
\tau^{\alpha \otimes \psi}\left(E_{S},\left\{f^{-1}\left(\mathcal{B}_{i}\right)\right\}\right)= & \tau^{\alpha \otimes \psi}\left(E_{L} \backslash \operatorname{int} T ;\left\{\mathcal{F}_{i}\right\}\right) \cdot \tau^{\alpha \otimes \psi}\left(E_{K} ;\left\{f^{-1}\left(\mathcal{D}_{i}\right)\right\}\right) \\
& \cdot \tau^{\alpha \otimes \psi}\left(\partial T,\left\{\mathcal{E}_{i}\right\}\right)^{-1} \\
\tau^{\alpha \otimes \psi}\left(E_{L},\left\{\mathcal{B}_{i}\right\}\right)= & \tau^{\alpha \otimes \psi}\left(E_{L} \backslash \operatorname{int} T ;\left\{\mathcal{F}_{i}\right\}\right) \cdot \tau^{\alpha \otimes \psi}\left(T ;\left\{\mathcal{D}_{i}\right\}\right) \cdot \tau^{\alpha \otimes \psi}\left(\partial T,\left\{\mathcal{E}_{i}\right\}\right)^{-1}
\end{aligned}
$$

(Here and in the next line the equalities of torsion are up to multiplication by an element of the form $\pm d h$ with $d \in \operatorname{det}(\alpha(\pi(L)))$ and $h \in H$.) Combining these results we now obtain that

$$
\tau^{\alpha \otimes \psi}\left(E_{S},\left\{f^{-1}\left(\mathcal{B}_{i}\right)\right\}\right)=\tau^{\alpha \otimes \psi}\left(E_{L},\left\{\mathcal{B}_{i}\right\}\right) \cdot \prod_{i=1}^{k} \Delta_{K}\left(z_{i}\right)
$$

This implies the desired equality.

7.2. Bing doubles. We consider the Bing double $B(K)$ of a knot $K$, in order to provide an example for a detailed computation. Note that if $K$ is a slice knot, then $B(K)$ is in fact a slice link (cf. Ci06, Section 1]). The converse is a well-known folklore conjecture.

Given any knot $K$ all the 'classical' sliceness obstructions (e.g. multivariable Alexander polynomial, Levine-Tristram signatures) of its Bing double are trivial (cf. [Ci06, Section 1] for a beautiful survey). It is therefore an interesting question to determine which methods and invariants detect the non-sliceness of Bing doubles. For interesting results, we refer to Cimasoni Ci06, Harvey Ha08, Cha Ch10. Ch09, and Cha-Livingston-Ruberman CLR08. In particular in CLR08 it was proved that if $K$ is not algebraically slice, $B(K)$ is not slice. Also it is known that there are algebraically slice knots with non-slice Bing doubles CK08, CHL08].

Now let $K=4_{1}$ be the Figure 8 knot. It is well-known that $K$ is not slice. For example this follows from FM66 since the Alexander polynomial $\Delta_{4_{1}}(t)=$ $t^{-1}-3+t$ is not a norm, namely not of the form $\pm t^{k} f(t) f\left(t^{-1}\right)$. In what follows we show that an appropriate twisted torsion invariant of the Bing double $B\left(4_{1}\right)$ is not a norm, and consequently $B\left(4_{1}\right)$ is not slice. (The fact that $B\left(4_{1}\right)$ is not slice had first been shown in Ch10 using the discriminant invariant.)

Let $x, y$ be the meridians of the two component unlink $L$ and denote by $A$ the unknotted curve in the definition of Bing doubles. It is well-known that $A$ represents 
the commutator $[x, y]$. We write $\xi=e^{2 \pi i / 8}$. Note that $\mathbb{Z}[\xi]$ is a UFD (cf. Wa97, Theorem 11.1]). Consider the representation $\alpha: \pi(L)=\langle x, y\rangle \rightarrow \mathrm{GL}(\mathbb{Z}[\xi], 8)$ given by

and

$$
\alpha(x)=\left(\begin{array}{cccccccc}
0 & 0 & 0 & \xi & 0 & 0 & 0 & 0 \\
1 & 0 & 0 & 0 & 0 & 0 & 0 & 0 \\
0 & 1 & 0 & 0 & 0 & 0 & 0 & 0 \\
0 & 0 & -i & 0 & 0 & 0 & 0 & 0 \\
0 & 0 & 0 & 0 & 0 & 0 & 0 & \xi \\
0 & 0 & 0 & 0 & -1 & 0 & 0 & 0 \\
0 & 0 & 0 & 0 & 0 & 1 & 0 & 0 \\
0 & 0 & 0 & 0 & 0 & 0 & -\xi & 0
\end{array}\right)
$$

$$
\alpha(y)=\left(\begin{array}{cccccccc}
0 & 0 & 0 & 0 & \xi & 0 & 0 & 0 \\
0 & 0 & 0 & 0 & 0 & 0 & 0 & -i \\
0 & 0 & 0 & 0 & 0 & 0 & \xi^{3} & 0 \\
0 & 0 & 0 & 0 & 0 & \xi^{7} & 0 & 0 \\
i & 0 & 0 & 0 & 0 & 0 & 0 & 0 \\
0 & 0 & 0 & \xi & 0 & 0 & 0 & 0 \\
0 & 0 & \xi^{3} & 0 & 0 & 0 & 0 & 0 \\
0 & -1 & 0 & 0 & 0 & 0 & 0 & 0
\end{array}\right) .
$$

It follows easily from Proposition 5.1 that $\alpha$ factors through a 2-group. We denote the representation $\pi_{1}\left(E_{B(K)}\right) \stackrel{f}{\rightarrow} \pi(L) \rightarrow \mathrm{GL}(\mathbb{Z}[\xi], 8)$ by $\alpha$ as well, and we let $\psi$ be the isomorphism $H_{1}\left(E_{B(K)}\right) \cong H_{1}\left(E_{L}\right) \rightarrow \mathbb{Z}^{2}$. (Here $K=4_{1}$.) We compute the eigenvalues of $\alpha(A)=\alpha([x, y])$ to be

$$
\left\{ \pm 1, \pm i, \pm e^{2 \pi i / 16}, \pm e^{2 \pi 3 i / 16}\right\} \text {. }
$$

We then calculate

$$
\begin{aligned}
\Delta_{K}(1) \cdot \Delta_{K}(-1) & \cdot \Delta_{K}(i) \cdot \Delta_{K}(-i) \\
& \cdot \Delta_{K}\left(e^{2 \pi i / 16}\right) \cdot \Delta_{K}\left(-e^{2 \pi i / 16}\right) \cdot \Delta_{K}\left(e^{2 \pi 3 i / 16}\right) \cdot \Delta_{K}\left(-e^{2 \pi 3 i / 16}\right)
\end{aligned}
$$

to equal 2115. If $B(K)$ was slice, then by Corollary 7.2 we would have

$$
2115= \pm d q \cdot \bar{q}
$$

for some $d \in \operatorname{det}(\alpha(\pi(L)))$ and for some $q$ in the quotient field of $\mathbb{Z}[\xi]$. Note that $q \bar{q}$ is a positive real number and note that $\operatorname{det}(\alpha(\pi(L))) \cap \mathbb{R}= \pm 1$. It therefore follows that if $B(K)$ is slice, then $2115=q \cdot \bar{q}$. Since $\mathbb{Z}[\xi]$ is a UFD we furthermore deduce that $q$ actually lies in $\mathbb{Z}[\xi]$. Now note that $2115=47 \cdot 45=47 \cdot(6+3 i)(6-3 i)$. Since $\mathbb{Z}[\xi]$ is a UFD we only have to show that 47 is not a norm. A direct calculation shows that any norm in $\mathbb{Z}[\xi]$ is of the form

$$
a^{2}+b^{2}+c^{2}+d^{2}+\sqrt{2}(a(b-d)+c(b+d))
$$

for some $a, b, c, d \in \mathbb{Z}$. One can now easily deduce that 47 is not a norm in $\mathbb{Z}[\xi]$. This concludes the proof of the claim.

Note that the process of Bing doubling can be iterated. We refer to the work of the first author Ch10], the first author and Kim [CK08, Cochran, Harvey and Leidy CHL08 and van Cott vCo09 for interesting results on iterated Bing doubling.

\section{REFERENCES}

[CS80] S. Cappell and J. Shaneson, Link cobordism, Comment. Math. Helv. 55 (1980), 20-49.

[Ch10] J. C. Cha, Link concordance, homology cobordism, and Hirzebruch-type defects from iterated p-covers, Journal of the European Mathematical Society 12 (2010), 555-610. 
[Ch09] J. C, Cha, Structure of the string link concordance group and Hirzebruch-type invariants, Indiana University Mathematics Journal 58 (2009), 891-928.

[CK08] J. C. Cha and T. Kim, Covering link calculus and iterated Bing doubles, Geometry and Topology 12 (2008), 2173-2201.

[CKo99] J. C. Cha and K. H. Ko, Signature invariants of links from irregular covers and nonabelian covers, Math. Proc. Cambridge Philos. Soc. 127 (1999), no. 1, 67-81.

[CKo06] J. C. Cha and K. H. Ko, Signature invariants of covering links, Trans. Amer. Math. Soc. 358 (2006), 3399-3412.

[CLR08] J. C. Cha, C. Livingston and D. Ruberman, Algebraic and Heegaard-Floer invariants of knots with slice Bing doubles, Math. Proc. Cam. Phil. Soc. 144 (2008), 403-410.

[Chp74] T. A. Chapman, Topological invariance of Whitehead torsion, Amer. J. Math. 96 (1974), no. 3, 488-497.

[Ci06] D. Cimasoni, Slicing Bing doubles, Algebr. Geom. Topol. 6 (2006), 2395-2415.

[CHL08] T. Cochran, S. Harvey and C. Leidy, Link concordance and generalized doubling operators, Algebr. Geom. Topol. 8 (2008), 1593-1646.

[CO90] T. Cochran and K. Orr, Not all links are concordant to boundary links, Bull. Amer. Math. Soc. (N.S.) 23 (1990), no. 1 (1990), 99-106.

[CO93] T. Cochran and K. Orr, Not all links are concordant to boundary links, Ann. of Math. (2) 138 (1993), no. 3, 519-554.

[COT03] T. Cochran, K. Orr and P. Teichner, Knot concordance, Whitney towers and $L^{2}-$ signatures, Ann. of Math. (2) 157 (2003), no. 2, 433-519.

[Du86] J. Duval, Forme de Blanchfield et cobordisme d'entrelacs bords, Comment. Math. Helv. 61 (1986), no. 4, 617-635.

[FM66] R. H. Fox and J. W. Milnor, Singularities of 2-spheres in 4-space and cobordism of knots, Osaka J. Math 3 (1966), 257-267.

[Fr05] S. Friedl, Link concordance, boundary link concordance and eta-invariants, Math. Proc. Cambridge Philos. Soc. 138 (2005), no. 3, 437-460.

[FK06] S. Friedl and T. Kim, Thurston norm, fibered manifolds and twisted Alexander polynomials, Topology, 45 (2006), 929-953.

[FK08] S. Friedl and T. Kim, Twisted Alexander norms give lower bounds on the Thurston norm, Trans. Amer. Math. Soc. 360 (2008), 4597-4618.

[FV09] S. Friedl and S. Vidussi, A survey of twisted Alexander polynomials, Preprint (2009).

[GL92] P. Gilmer and C. Livingston, The Casson-Gordon invariant and link concordance, Topology 31 (1992), 475-492.

[Go78] C. McA. Gordon, Some aspects of classical knot theory, Knot theory (Proc. Sem., Planssur-Bex, 1977), Lecture Notes in Math. 685 (1978), 1-65.

[Ha08] S. Harvey, Homology cobordism invariants and the Cochran-Orr-Teichner filtration of the link concordance group, Geom. Topol. 12 (2008), 387-430.

[HKL08] C. Herald, P. Kirk and C. Livingston, Metabelian representations, twisted Alexander polynomials, knot slicing, and mutation, Preprint (2008), to appear in Math. Z.

[Ka77] A. Kawauchi, On quadratic forms of 3-manifolds, Invent. Math. 43 (1977), 177-198.

[Ka78] A. Kawauchi, On the Alexander polynomials of cobordant links, Osaka J. Math. 15 (1978), no. 1, 151-159.

[KL99a] P. Kirk and C. Livingston, Twisted Alexander invariants, Reidemeister torsion and Casson-Gordon invariants, Topology 38 (1999), no. 3, 635-661.

[KL99b] P. Kirk and C. Livingston, Twisted knot polynomials: inversion, mutation and concordan, Topology 38 (1999), no. 3, 663-671.

[Ko85] K. H. Ko, Algebraic classification of simple links of odd dimension $\geq 3$, Unpublished (1985).

[Ko87] K. H. Ko, Seifert matrices and boundary link cobordism, Trans. Amer. Math. Soc. 299 (1987), 657-681.

[Le94] J. Levine, Links invariants via the eta invariant, Comm. Math. Helv. 69 (1994), 82-119.

[Le07] J. Levine, Concordance of boundary links, Journal of Knot Theory and its Ramifications 16 (2007), no. 9, 1111-1120.

[Lia77] C. Liang, An algebraic classification of some links of codimension two, Proc. Amer. Math. Soc. 67 (1977), 147-151.

[Liv09] C. Livingston, The concordance genus of a knot, II, Algebraic \& Geometric Topology 9 (2009), 167-185.

[Mi57] J. Milnor, Isotopy of links, Algebraic geometry and topology, A symposium in honor of S. Lefschetz, 280-306. Princeton University Press, Princeton, N. J. (1957).

[Mi62] J. Milnor, A duality theorem for Reidemeister torsion, Ann. of Math. (2) 76 (1962), $137-147$.

[Mi66] J. Milnor, Whitehead torsion, Bull. Amer. Math. Soc. 72 (1966), 358-426. 
[Mi87] W. Mio, On boundary-link cobordism, Math. Proc. Camb. Phil. Soc. 101 (1987) 259-266.

[Mu67] K. Murasugi, On a certain numerical invariant of link types, Trans. Amer. Math. Soc. 117 (1967), 387-422

[Na78] Y. Nakagawa, On the Alexander polynomials of slice links, Osaka J. Math. 15 (1978), no. 1, 161-182.

[Sh03] D. Sheiham, Invariants of boundary link cobordism, Mem. Amer. Math. Soc. 165, no. 784 (2003).

[Sh06] D. Sheiham, Invariants of boundary link cobordism. The Blanchfield-Duval Form, Noncommutative Localization in Algebra and Topology, LMS Lecture Notes Series 330 (2006), Cambridge University Press, 143-219.

[Sm89] L. Smolinsky, Invariants of link cobordism, Proceedings of the 1987 Georgia Topology Conference (Athens, GA, 1987). Topology Appl. 32 (1989), no. 2, 161-168.

[Sta65] J. Stallings, Homology and central series of groups, Journal of Algebra 2 (1965), 170-181.

[Str74] R. Strebel, Homological methods applied to the derived series of groups, Comment. Math. Helv. 49 (1974), 302-332.

[Ta02] A. Tamulis, Knots of Ten or Fewer Crossings of Algebraic Order Two, J. Knot Theory Ramifications 11 (2002), no. 2, 211-222.

[Tu86] V. Turaev, Reidemeister torsion in knot theory, Russian Math. Surveys 41 (1986), 119182.

[Tu01] V. Turaev, Introduction to Combinatorial Torsions, Lectures in Mathematics, ETH Zürich, 2001.

[Tu02] V. Turaev, Torsions of 3-manifolds, Progress in Mathematics 208. Birkhauser Verlag, Basel, 2002.

[vCo09] C. Van Cott, An obstruction to slicing iterated Bing doubles, Preprint (2009).

[Vo90] P. Vogel, Representations of links groups, KNOTS 90: Proceedings of Osaka knot theory conference (1990), W. de Gruyter, Berlin, 381-388.

[Wa97] L. Washington, Introduction to cyclotomic fields, Second edition. Graduate Texts in Mathematics, 83. Springer-Verlag, New York (1997).

[We03] S. Weintraub, Representation Theory of Finite Groups: Algebra and Arithmetic, AMS Graduate Studies in Mathematics (2003).

Pohang University of Science and Technology, Pohang Gyungbuk, Republic of Korea

E-mail address: jccha@postech.ac.kr

UNIVERSity OF WARWICK, COVEntry, UK

E-mail address: s.k.friedl@warwick.ac.uk 